\title{
Nanoprobing of semiconductor heterointerfaces: quantum dots, alloys and diffusion
}

\author{
R S Goldman \\ Department of Materials Science and Engineering, University of Michigan, Ann Arbor, \\ MI 48109-2136, USA
}

Received 19 April 2004

Published 16 June 2004

Online at stacks.iop.org/JPhysD/37/R163

doi:10.1088/0022-3727/37/13/R01

\begin{abstract}
Interfaces play a key role in the performance of electronic, optoelectronic and photovoltaic devices. Within epitaxial semiconductor heterostructures, interfaces are commonly characterized using various methods including transmission electron microscopy, secondary ion mass spectroscopy, $\mathrm{x}$-ray diffraction, photoluminescence spectroscopy and capacitance-voltage profiling. The averaging present in these conventional techniques limits their ability to resolve critical atomic-scale features. In addition, many of these methods require detailed modelling in order to determine quantitative interface profiles. Thus, these techniques are often not suitable for obtaining the localized information needed to elucidate the structure and properties of heterointerfaces. Alternatively, nanoprobing of heterointerfaces using cross-sectional scanning tunnelling microscopy (XSTM) has emerged as a powerful method for resolving atomic features at interfaces within heterostructures. In this paper, we describe XSTM and discuss its application to several important issues in semiconductor heterostructure materials, including the formation and ordering of quantum dot arrays, direct measurements of interdiffusion and segregation lengths and investigations of the mechanisms of alloy phase separation.
\end{abstract}

\section{Introduction}

In the past few years, cross-sectional scanning tunnelling microscopy (XSTM) has emerged as a powerful tool for resolving atomic-scale features at interfaces within heterostructures [1,2]. For XSTM, a cross-section is prepared by cleaving the structure in ultra-high vacuum (UHV) to expose an atomically flat surface. STM is then performed on this cleaved surface. Since the cleaved surface is generally atomically flat, with monolayer height steps spaced several hundreds of nanometres apart, the apparent topographic contrast observed in constant-current images is primarily due to variations in the electronic properties of the relevant layers. In cases where the cleaved surface does not reconstruct, it is possible to obtain atomic-scale information about the bulk-like structure, chemistry and electronic properties of the layers.
The major advantage of XSTM for resolving atomic-scale features over other techniques, including TEM, is that the images collected are primarily associated with the top layer of the cleaved surface, rather than an average over many layers. In addition, flat cleaves generally occur over hundreds of $\mu \mathrm{m}^{2}$; therefore, XSTM enables resolution of atomic-scale features over macroscopic length-scales.

In this paper, we describe XSTM and discuss its application to investigations of semiconductor heterointerfaces, with an emphasis on epitaxial semiconductor heterostructures. We present several recent examples, including studies of the formation and ordering of quantum dot (QD) arrays, direct measurements of interdiffusion and segregation lengths and investigations of the mechanisms of alloy phase separation. We also discuss several future directions, including studies of Mn segregation in GaAs and InAs/GaAs QDs, as 
well as studies of interface formation in (110)-oriented layered III-V heterostructures.

\section{Cross-sectional scanning tunnelling microscopy}

For the most common application of XSTM, a cross-section of an (001)-oriented layered III-V compound semiconductor heterostructure is prepared by cleaving the structure in UHV to expose an atomically flat (110) surface (as shown in figure 1). Typically, constant-current STM is then performed on this cleaved surface. Since the cleaved surface is generally atomically flat, with monolayer steps spaced hundreds of nanometres apart, the apparent topographic contrast observed in constant-current images is primarily due to variations in the electronic properties of the relevant layers. Furthermore, the UHV-cleaved (110) surface of most III-V compounds does not reconstruct, and the dangling bond states do not lie within the energy band gap [3]. Thus, it is possible to obtain information about the bulk-like structure, chemistry and electronic properties of the layers. We note that it is possible to perform XSTM on ambient-cleaved (110) surfaces of III-V compound semiconductor heterostructures by a chemical passivation of the dangling bond surface states [2]. Similarly, for both ambient-cleaved and UHVcleaved (110) and (111) surfaces of group IV elemental semiconductor heterostructures, successful XSTM requires chemical passivation of the dangling bond surface states [4-6].

Figure 1 shows a schematic of XSTM applied to a compound semiconductor superlattice (SL). The apparent topographic contrast shown in figure 1 is due to differences in the densities of electronic states of the two constituent epilayers of the SL. The constituent epilayer with the lower density of states appears dark because the tip moves towards the surface in order to maintain a constant tunnelling current. Correspondingly, the constituent epilayer with the higher density of states appears bright because the tip moves away from the surface in order to maintain a constant tunnelling current. Figure 2 shows schematic energy diagrams for empty state imaging of the two constituent epilayers of the SL in figure 1. In both figures $2(a)$ and $(b)$, application of a constant

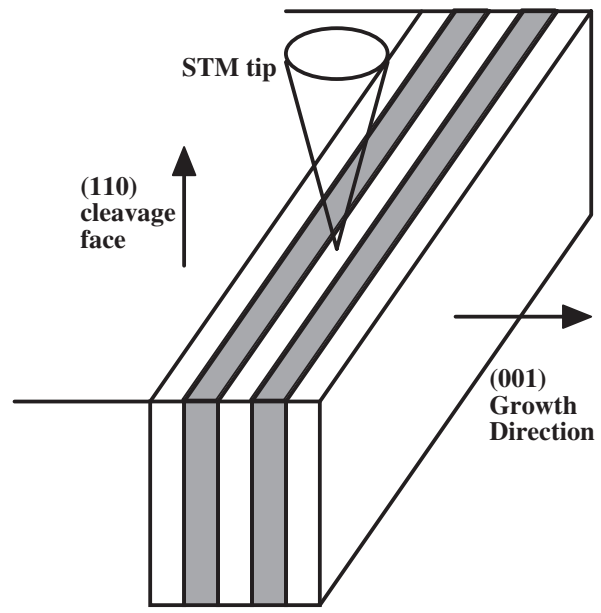

Figure 1. Schematic of XSTM applied to a semiconductor SL. For example, the alternating bright and dark layers might correspond to $\mathrm{AlAs}$ and GaAs layers, respectively. sample bias voltage, $V$, results in electrons tunnelling from the tip into the empty conduction band states. For most positive sample bias voltages, the smaller band gap GaAs has more empty states for the electrons to tunnel into than the larger band gap AlAs. Thus, GaAs and AlAs layers have higher and lower densities of empty states, and therefore appear bright and dark in the constant-current XSTM image.

It is also possible to obtain spectroscopic information about semiconductor heterostructures using conductance imaging $(\mathrm{d} I / \mathrm{d} V)$ and variable tip-sample separation spectroscopy. In variable tip-sample spectroscopy, the tunnelling current is measured while both the bias voltage and the tip-sample separation are varied in a controlled manner [7]. This method increases the dynamic range of the tunnelling current (particularly in the vicinity of the band gap), thereby enabling accurate determination of the energetic positions of the band edges.

\section{Self-assembled QD SLs}

\subsection{Introduction}

Recently, arrays of self-assembled QD SLs have shown significant promise for a wide range of electronic and optoelectronic device applications. Dense arrays of uniformly sized QDs are required for most device applications. Meanwhile, variations in the size and shape of such QDs significantly impact their electronic structure and luminescence properties. Hence, the nanometre-scale details of the uniformity of dot organization, as well as the evolution of dot shapes and sizes, are crucial for the development of novel applications. A number of recent XSTM studies have focused on some of these issues in both InAs and InGaAs QDs [8-17].

In general, self-assembled QD formation is driven by the elastic relaxation of stress via island nucleation. The vertical stacking of QDs is often explained by the preferred nucleation of islands at strain energy minima directly above buried islands $[18,19]$. However, the mechanisms of lateral ordering of QD arrays are the subject of continued debate [19-22]. In addition, the perfection and stability of QD arrays are often determined by additional processes, such as diffusion and segregation, occurring during epitaxial growth and subsequent thermal annealing [16,23]. Therefore, we have investigated the evolution and lateral ordering of a series of multi-period InAs/GaAs QD SLs, annealed for various times. XSTM studies reveal that the average spacing between dot
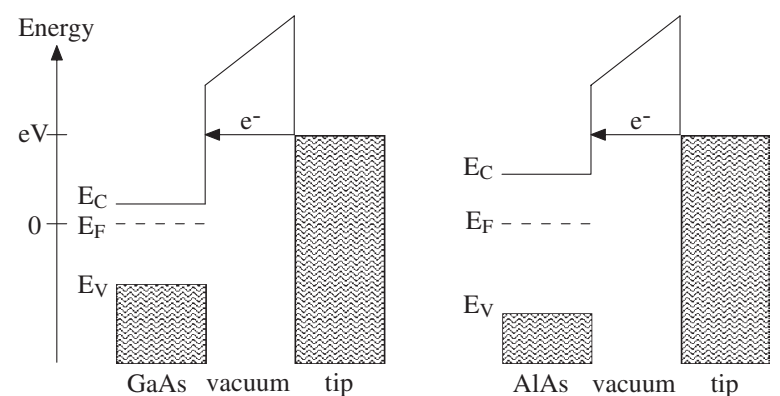

Figure 2. Schematic of the tunnelling process for a positive sample bias voltage. Electrons tunnel from the STM tip into the empty conduction band states of either $(a) \mathrm{GaAs}$ or $(b)$ AlAs. 
(a)

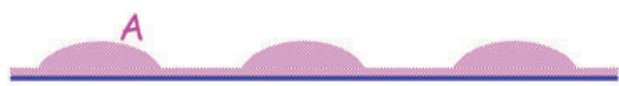

B

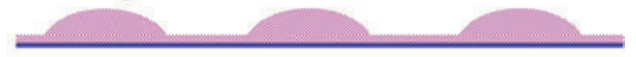

(b)

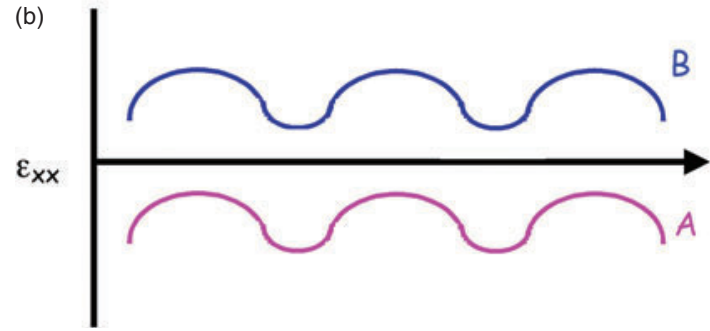

Figure 3. Schematic of the dot stacking process for a compressively stressed system, A/B. (a) SLs of 'A' dot layers and 'B' spacer layers; (b) corresponding in-plane stress as a function of distance, in both 'A' and 'B' layers.

(This figure is in colour only in the electronic version)

columns increases with increased annealing. Interestingly, the spacing between dot columns is constant throughout the thickness of a particular dot SL, and the trend of increasing dot column spacing with annealing time depends on the sequence of SL growth. Together, these results suggest a new conceptual model for QD ordering in which surface nucleation during growth leads to the initial placement of dots, and strain-enhanced bulk diffusion leads to the dissolution of indium atoms from existing dots into the wetting layer. This conceptual model is likely to be applicable to a wide variety of heteroepitaxial systems.

\subsection{Nucleation model for self-ordering of $Q D s$}

The Tersoff nucleation model for self-ordering of QD SLs explains the vertical stacking of QDs via the surface strain field resulting from a buried strained island [19]. Figure 3 shows an example of QDs of material 'A' grown on a substrate 'B', where the lattice parameter of 'A' is larger than that of 'B'. Since the compressive strain in 'A' is partially elastically relaxed by the formation of the QDs, a lateral strain variation develops at the surface. As the spacer ' $\mathrm{B}$ ' is deposited on top of ' $\mathrm{A}$ ', the surface develops a similar lateral strain variation, with the opposite sign. For appropriately chosen spacer thicknesses, the strain variation in ' $\mathrm{B}$ ' consequently determines the placement of the next 'layer' of QDs of 'A'. In effect, regions of higher tensile (compressive) strain favour the attachment of large (small) atoms.

Tersoff's nucleation model also predicts that the growth of additional periods of QD SLs will result in an increasing regularity of the spacings between the QDs (as shown in figure 4). In the case where widely spaced islands evolve into closely spaced columns of stacked QDs, this would necessarily occur through the merging of 'columns' of QDs, as has been reported in some cases. Furthermore, there would be a sort of 'differential' spacing between QD 'columns' as one moves upwards within a QD SL. Although this model appears to explain some experimental data $[12,22]$, there are many situations for which the model needs to be modified, as will be discussed below.

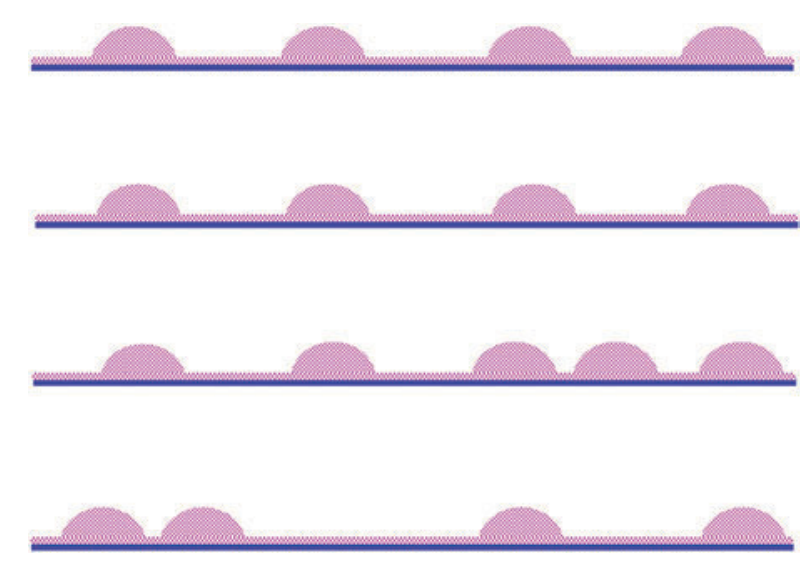

Figure 4. Schematic of the nucleation model for ordering of stacked dots for a compressively stressed system, A/B. Initially, islands are nucleated in random positions. As additional spacer layers and dot layers are deposited, islands are nucleated both above and between buried islands. After the process is repeated several times, the spacing between the islands becomes regular.

(This figure is in colour only in the electronic version)

\subsection{Experiment}

Sequences of multi-period QD SLs consisting of 2.6 ML InAs and $5 \mathrm{~nm}$ GaAs were grown by solid-source molecular beam epitaxy (MBE) on Si-doped $\left(n \sim 3 \times 10^{18} \mathrm{~cm}^{-3}\right.$ ) (001)-oriented GaAs substrates. Sequences of 1-, 5-, 10and 20-period and 5-, 5-, 5- and 5-period QD SLs were grown, and will be referred to as '1-5-10 dot SLs' [14] and '5-5-5 dot SLs' [24], respectively. In each case, in order to prevent the interaction of strain fields from different SLs, each layer of SLs was separated by a $140 \mathrm{~nm}$ multilayer consisting of a $40 \mathrm{~nm}$ total thickness of $\mathrm{AlAs} / \mathrm{GaAs}$ short-period SL sandwiched between two $50 \mathrm{~nm}$ GaAs layers, which we refer to as an isolation layer. The QD SLs and AlAs/GaAs shortperiod SLs were grown at $510^{\circ} \mathrm{C}$ and $620-660^{\circ} \mathrm{C}$, respectively. The growth rates for AlAs, GaAs and InAs were $0.9 \AA \mathrm{s}^{-1}$, $2.0 \AA \mathrm{s}^{-1}$ and $0.3 \AA \mathrm{s}^{-1}$, respectively. We note that for these relatively slow AlAs growth rates, surface roughening is generally avoided. Before (after) each set of SLs, the substrate temperature was increased (decreased) over the thickness of the $50 \mathrm{~nm}$ GaAs layer, and the AlAs/GaAs short-period SLs were grown at $620-660{ }^{\circ} \mathrm{C}$. Thus, the first, second and third layers of dot SLs were effectively in situ annealed at $620-660{ }^{\circ} \mathrm{C}$ for $22.2 \mathrm{~min}, 14.8 \mathrm{~min}$ and $7.4 \mathrm{~min}$, respectively.

\subsection{InAs/GaAs QD SLs}

A large-scale XSTM topographic image showing 5- and 10-period InAs/GaAs QD SLs within the 1-5-10 dot SLs is shown in figure 5. Within each layer of dot SLs, bright ellipses of InAs QDs sandwiched between darker layers corresponding to GaAs are apparent. It is evident from the image that the 5- and 10-period SLs have organized into columns of vertically correlated InAs QDs. Although the column axis is primarily oriented along the [001] growth direction, it is occasionally rotated towards the [110] or [ $\overline{1} \overline{1} 0]$ direction, producing 'leaning towers' of QDs. In some cases, the normal of an individual QD within a column is rotated up to $11^{\circ}$ away from the [001] direction (as shown in figure 6(a)). In other cases, a 


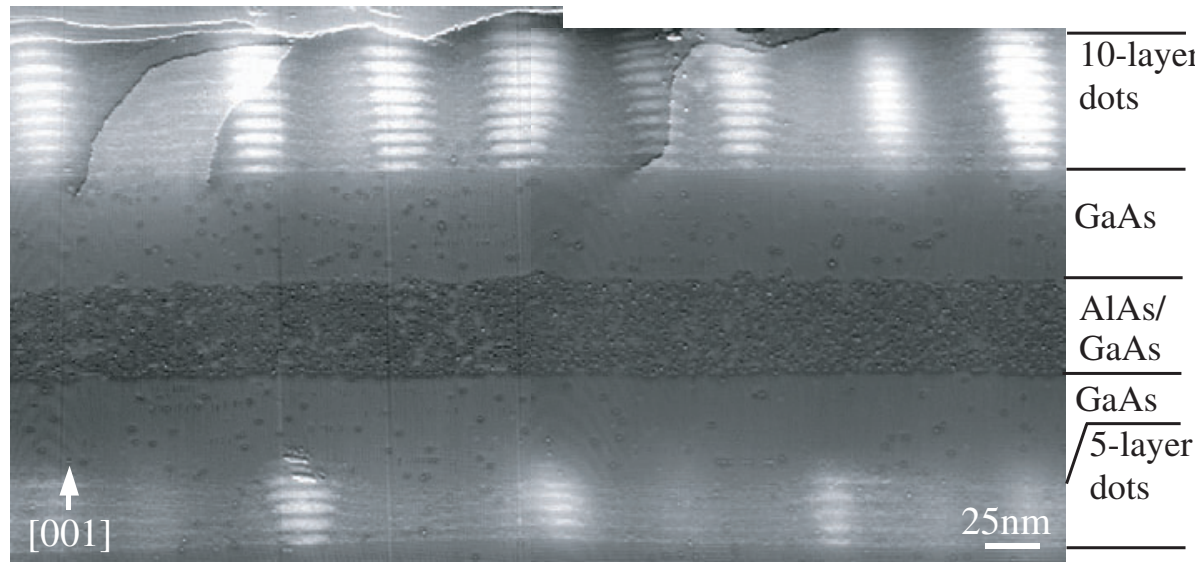

Figure 5. Large-scale XSTM topographic image of low $T$ annealed QD SLs. The image was acquired at a sample bias voltage of $-2.5 \mathrm{~V}$. The grey-scale range displayed is $13.6 \AA$.

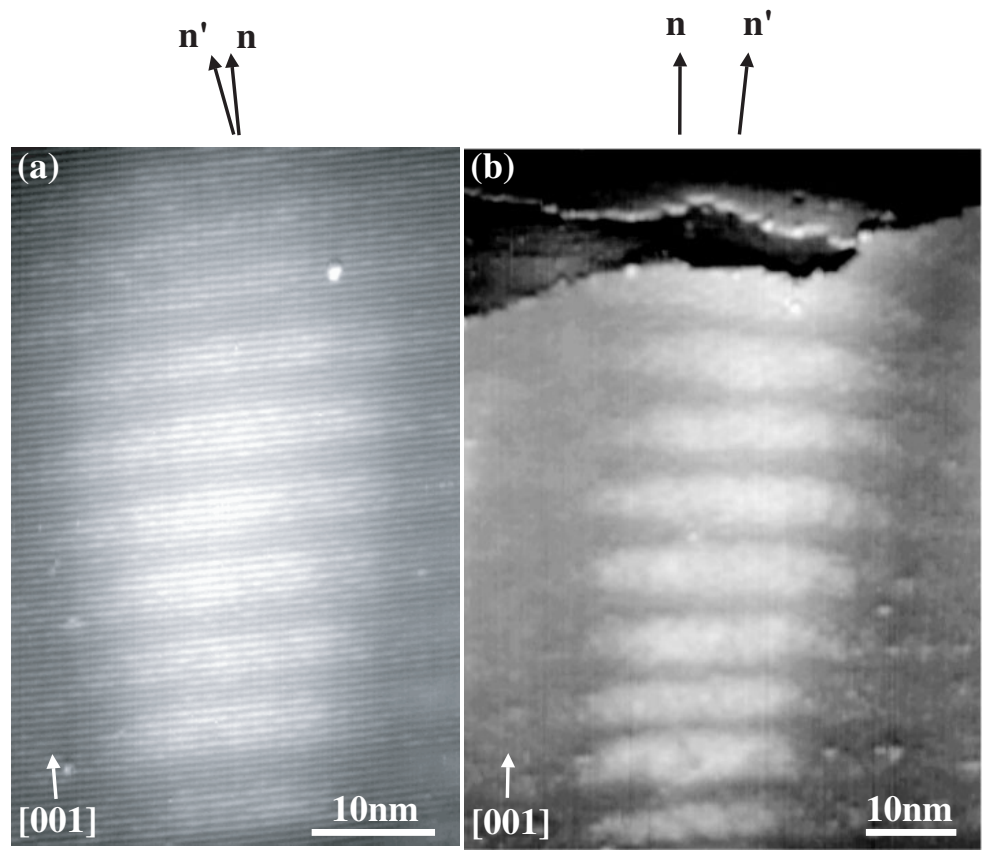

Figure 6. High-resolution XSTM topographic images of 10-period low $T$ annealed QD SLs, obtained at sample bias voltages of $-2.2 \mathrm{~V}$. For both $(a)$ and $(b), n$ and $n^{\prime}$ are the normals of the bottom and top dots in the column, respectively. In $(a), n^{\prime}$ is rotated from the [001] direction towards the [110] direction. In $(b), n^{\prime}$ is shifted laterally towards the [110] direction.

misalignment in the vertical correlation of QDs has occurred (as shown in figure $6(b)$ ). In general, the apparent diameters of the QDs increase monotonically along the [001] growth direction. This increase in the apparent QD diameter is balanced by the depletion of indium from the wetting layers located between the vertical columns of QDs. Similar results were obtained for the 5-5-5 dot SLs.

It is interesting to note that for both the 1-5-10 and the 5-5-5 dot SLs, the spacing between dot columns appears to decrease for successive layers of dot SLs, although the lateral positions of dot columns between the various layers are not strictly correlated or anticorrelated, consistent with the effective separation of strain fields by the isolation layers. We note that GaAs layers of comparable thicknesses have been shown to effectively isolate the strain fields between successive layers of InAs/GaAs QDs [25].
Since the spacing between dot columns appears to be fixed throughout the thickness of a particular dot SL, this cannot be explained by a simple nucleation-based mechanism, such as the Tersoff model discussed earlier. Alternatively, these results suggest that significant In diffusion has occurred during annealing. Since the dot column spacings and the dot dimensions are significantly larger than recently measured In-Ga interdiffusion lengths $[15,16]$, it is likely that the diffusion process has been enhanced by strain.

In our case, it essentially seems as if the QDs somehow knew that they were going to be part of a 1-, 5- or 10-period QD SL, which is not realistic. Thus, we consider two possible models, which rely upon a combination of surface nucleation and bulk diffusion. Surface nucleation during growth leads to the initial placement of the QDs, and bulk diffusion during annealing presumably leads to the rearrangement of 
(a)

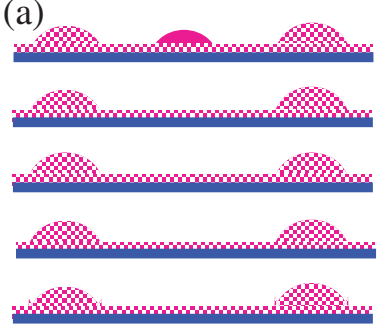

(b)

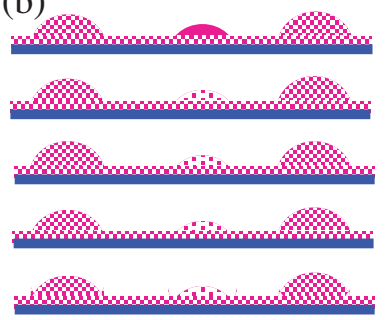

(c)

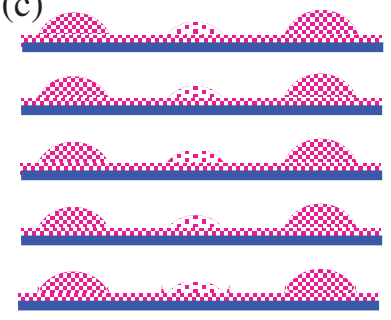

(d)

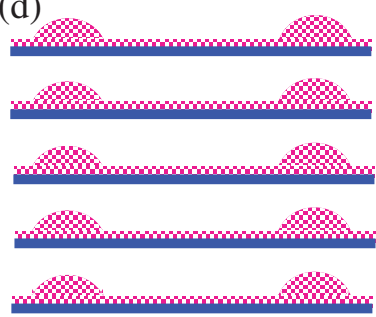

Figure 7. Schematics of $(a)-(b)$ 'nucleation plus agglomeration' and $(c)-(d)$ 'nucleation plus dissolution' models: $(a)$ nucleation of an additional small dark pink dot, followed by $(b)$ agglomeration of indium atoms from wetting layer forming additional light pink dots; $(c)$ dot columns formed during the growth process, followed by $(d)$ dissolution of indium atoms into the wetting layer.

indium atoms, either by the agglomeration of indium atoms from the wetting layer to form additional QDs ("nucleation plus agglomeration') or by the dissolution of indium atoms into the wetting layer from existing QDs ('nucleation plus dissolution').

The proposed nucleation plus diffusion mode are shown in figures 7(a) and $(b)$. Note that the differences in the sizes and colours of the dots are meant to distinguish them, rather than implying some explicit difference in their sizes. In the 'nucleation plus diffusion' mechanism, one would assume that somewhere beyond 5 dot SL periods, there is sufficient strain to warrant the nucleation of an additional dot, shown as a smaller pink dot in figure 7(a). This would appear to be consistent with the Tersoff model. However, in order to be consistent with our experimental observations, an additional step must occur. The additional step (shown in figure $7(b)$ ) is the agglomeration of indium atoms from the wetting layer to form an additional QD. Thus, the additional smaller pink dot acts as a stressor, enhancing the driving force for the agglomeration of indium atoms. The possibility for agglomeration of indium atoms from the wetting layer is supported by our recent report of significant In-In pair correlations within the wetting layers of InAs/GaAs QDs [17]. In the 'nucleation plus dissolution' mechanism, the QDs shown in figure 7(c) are produced during the growth process. Upon annealing, the middle column of QDs dissolves into the wetting layer, leading to a wider spacing between QD columns (as shown in figure $7(d)$ ). For this conceptual model, the relative spacing between successive layers of QD SLs would depend only on the differences in their annealing times.

In order to determine the relative roles of agglomeration and dissolution, as well as the effect of strain on the diffusion process determining QD column spacings, we quantitatively compared the effects of annealing on the spacing between QD columns in the 1-5-10 and the 5-5-5 QD SLs. To quantify the lateral spacing between the QD columns, we used a column counting criterion as follows. Lateral tip height profiles (line-cuts) averaged over the thickness of potential columns of QDs were determined. From the averaged linecuts, protrusions with maximum tip heights greater than $2 \AA$ above the GaAs background were considered dot columns. For both the 1-5-10 and 5-5-5 dot SLs, we used several large-scale XSTM images, spanning a total length of more than $12 \mu \mathrm{m}$ and $20 \mu \mathrm{m}$, respectively. In both cases, we find a general trend of an increase in average lateral column spacing with increasing annealing time. In addition, we define a 'scaled column spacing' as the ratio of the average column spacing,

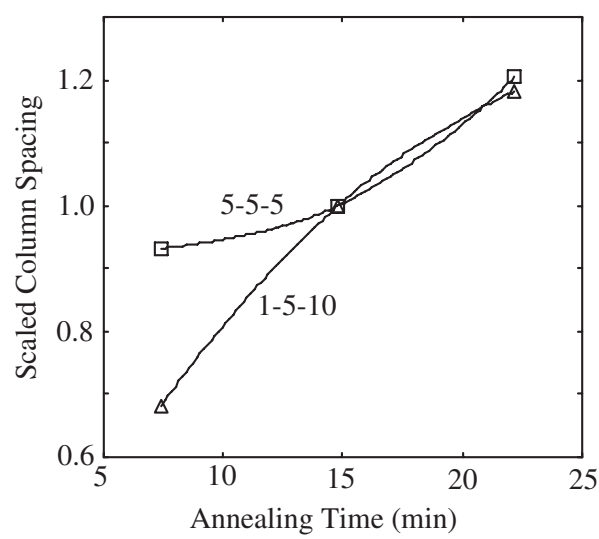

Figure 8. Scaled column spacing plotted against annealing time for the 1-, 5- and 10-period and 5-, 5- and 5-period QD SL. The lines are meant as a guide to the eye. (Reprinted from [24].)

after a particular annealing time, to the average column spacing for the 5-period SL annealed for $14.8 \mathrm{~min}$. In figure 8, we plot the scaled column spacing against the annealing time for both the 5-5-5 and 1-5-10 QD SLs. As the scale column spacing increases (decreases), the dot columns are further apart (closer together). For both the 5-5-5 and 1-5-10 QD SLs, the dot columns are further apart as the annealing time is increased; thus, the scaled column spacing increases with annealing time.

If the 'nucleation plus agglomeration' model (shown in figures $7(a)$ and $(b)$ ) were the predominant QD ordering mechanism, the scaled column spacing of the 1-5-10 QD SL would decrease with sequential dot layers, as is observed. On the other hand, the scaled column spacing of the 5-5-5 QD SL would either increase slightly or remain constant with sequential QD layers. Since the scaled column spacing decreases with sequential dot layers for both the 1-5-10 and 5-5-5 QD SLs, the 'nucleation plus agglomeration' model is considered unlikely to be the mechanism of QD ordering.

Alternatively, since the scaled column spacing increases with annealing time for both the 1-5-10 and 5-5-5 dot SLs, the QD ordering is likely to be governed by the "nucleation plus dissolution' model (shown in figures $7(c)$ and $(d)$ ). This is further supported by a close examination of the 5-period QD SL displayed in the image of figure 3, which reveals faint indications of additional columns of vertically correlated InAs islands between the brightest QD columns. The apparent columns do not satisfy the height criterion for column counting discussed above, and may be due to columns of larger islands whose central axes are located well below the cleaved surface. 
Alternatively, the columns are located in the same planes as the others, but they have started to dissolve, such that indium has dissolved into the wetting layer (as illustrated in figure $7(d)$ ). As will be discussed later, the dissolution of InAs islands is presumably driven by the residual strain present in the QD SLs. In essence, the diffusion may provide a means of relaxing strain.

It is interesting to note that the effect of increasing column spacing with annealing time is more significant for the 1-5-10 dot SLs in comparison with the 5-5-5 dot SLs. Although the sequential layers of 5-5-5 dot SLs have essentially identical stress states, the sequential layers of 1-5-10 QD SLs are likely to have significantly different stress states. For example, the 10-period QD columns often appear to contain screw components of $60^{\circ}$ dislocations, suggesting the presence of significant plastic relaxation, and consequently much less residual strain [15]. Interestingly, the plastically relaxed 10-period dot SLs have a reduced scaled column spacing in comparison with the 5-period QD SLs annealed for the same amount of time. Thus, it is likely that strain is playing a significant role in the diffusion process which ultimately determines the lateral spacing between QD columns.

\subsection{Summary and conclusions}

In summary, we have used XSTM to investigate the mechanisms of lateral ordering of InAs/GaAs QD SLs. We determined the lateral spacing between dot columns in a series of multiple-period QD SLs annealed for a variety of times. We find that the average column spacing increases and the distribution of spacings is broadened as a function of annealing time. Taken together, these results suggest that the lateral column spacing is determined by surface nucleation during growth followed by strain-enhanced island dissolution during annealing.

\section{Interdiffusion and segregation}

\subsection{Introduction}

Diffusion and segregation are fundamental processes of critical importance for the design of electronic and optoelectronic devices based on semiconductor heterostructures. Direct measurements of interdiffusion and segregation lengths are generally limited by the inherent averaging which occurs in conventional characterization techniques including transmission electron microscopy and x-ray diffraction. Most measurements of interdiffusion lengths in III-V systems have been achieved using photoluminescence spectroscopy, where interpretation of the data relies on several assumptions regarding the alloy composition and lateral uniformity, interface abruptness, heterojunction band-offsets, positions of confined states, etc. Furthermore, most analyses assume the applicability of Fick's first law, in which case the diffusional flux is driven solely by a concentration gradient, without contributions from additional potential gradients. In this section, we describe a novel method for measuring interdiffusion and segregation lengths, which involves the application of XSTM to QD SLs which consist of nearly three-dimensional arrays of QDs. Although these nearly three-dimensional arrays of QDs are highly ordered, there are regions between the QDs which contain sparse concentrations of individual atoms which have not agglomerated to form a QD. For example, in the InAs/GaAs system, the regions between the QDs contain a few layers of sparsely populated In atoms within a GaAs matrix. We describe $\mathrm{In} / \mathrm{Ga}$ atom counting using XSTM, leading to the first direct atomlevel measurements of In-Ga interdiffusion and In surface segregation lengths [14]. We also examine the applicability of Fick's first law to this system, considering the possibility of lateral In segregation occurring simultaneously with vertical In-Ga interdiffusion. We determine lateral In segregation via the quantification of lateral In-In pair correlations within the wetting layers, followed by a comparison with randomly generated In-In pair distributions [17].

\subsection{Experiment}

For these investigations, QD SLs were grown by solid-source molecular beam epitaxial growth, as described in an earlier report [15]. Each period of the InAs/GaAs QD SLs consisted of 2.6 monolayers of InAs, followed by $5 \mathrm{~nm} \mathrm{GaAs}$, with a $10 \mathrm{~s}$ interrupt under $\mathrm{As}_{4}$ flux separating their growth at $510{ }^{\circ} \mathrm{C}$. The QDs were effectively in situ annealed at $620^{\circ} \mathrm{C}$ for $22.5 \mathrm{~min}$. Ex situ post-growth rapid thermal annealing was performed in argon gas at $900^{\circ} \mathrm{C}$ for $30 \mathrm{~s}$. We will refer to the QD SL before and after $e x$ situ annealing cycles as low $T$ and high $T$ annealed wetting layers, respectively.

\subsection{In-Ga interdiffusion and In surface segregation}

Figures $9(a)$ and $(c)$ show high-resolution XSTM images in the vicinity of the low $T$ and high $T$ annealed 1-period InAs/GaAs SLs. In the darker regions of these images, fringes with a spacing of $5.65 \AA$, corresponding to the (001) lattice planes of GaAs, are observed. Since these images were acquired with a positive sample bias voltage (empty state images), the cation sublattice is resolved and the bright spots primarily correspond to In atoms in a GaAs matrix [26,27]. However, some of these bright spots might also be due to point defects including $\mathrm{Si}_{\mathrm{Ga}}$ and $\mathrm{V}_{\mathrm{Ga}}$. Many atomic-resolution images show a point defect concentration of approximately $3 \times 10^{18} \mathrm{~cm}^{-3}$, consistent with the targeted doping concentration in these structures. This point defect concentration is significantly less than the indium concentration, which is approximately $2 \times 10^{20} \mathrm{~cm}^{-3}$ for every $1 \%$ of In substituting at a Ga site.

After high $T$ annealing, significant intermixing is observed in the wetting layers. Figures $9(a)$ and $(c)$ show regions of a wetting layer in the vicinity of an island for the low $T$ and high $T$ annealed 1-period SLs, respectively. It is evident from these images that the high $T$ annealing has significantly increased the vertical extent of the wetting layer. The spatial distribution of In atoms was obtained from an analysis of several high-resolution XSTM images, spanning areas greater than $2000 \mathrm{~nm}^{2}$ for the low $T$ and high $T$ annealed wetting layers, respectively [14]. In each image, we used an algorithm to identify In and Ga atoms in bilayers within $12 \mathrm{~nm}$ of the atomic row with maximum In concentration. We estimated a tip height criterion of $0.85 \pm 0.05 \AA$ with respect to the GaAs background (as shown in figure $9(b)$ ). 


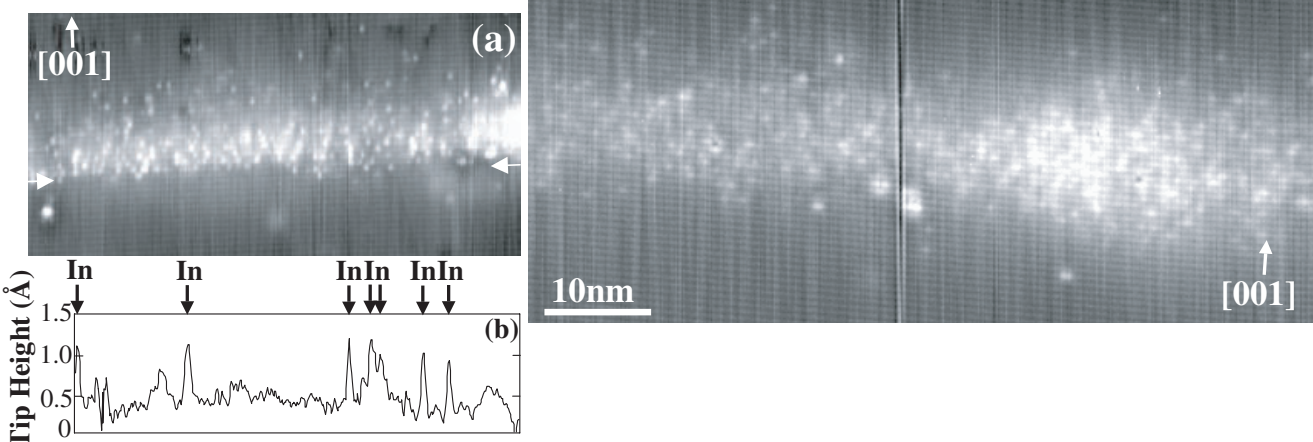

Figure 9. Comparison of atomic-resolution images of the wetting layers in single-period (a) low $T$ and (c) high $T$ annealed InAs/GaAs QD SLs. The images were acquired at sample bias voltages of $(a)+2.5 \mathrm{~V}$ and $(c)-2.4 \mathrm{~V}$. The grey-scale ranges displayed are $(a) 3.1 \AA$ and (c) $2.4 \AA$. A cut along the line indicated by arrows in $(a)$ is displayed in $(b)$. Pixels with tip height of $0.85 \pm 0.05 \AA$ above the GaAs background were considered to be In atoms and are marked by the downward pointing arrows in $(b)$.

In figure 10(a), for both the low $T$ and high $T$ annealed wetting layers, the fraction of indium sites occupied by indium, $f_{\text {In }}$, is plotted as a function of the number of bilayers, with the origin set artificially at that layer with the largest $f_{\text {In }}$. For both the low $T$ and high $T$ annealed wetting layers, the uncertainty in measuring $f_{\text {In }}$ due to possible contributions from point defects including $\mathrm{Si}_{\mathrm{Ga}}$ or $\mathrm{V}_{\mathrm{Ga}}$ is less than 0.014 , the point size in figure $10(a)$. It is evident from the plot that the low $T$ annealed $f_{\text {In }}$ is not symmetrical about the origin. On the substrate side of the origin, $f_{\text {In }}$ apparently fits a Gaussian, the thin film solution of the diffusion equation. The high $T$ annealed $f_{\text {In }}$ is more symmetric about the origin, and also apparently fits a Gaussian. For both the low $T$ and high $T$ annealed wetting layers, the concentration of indium atoms is expressed as $C_{\text {In }}=100 \times f_{\text {In }}$ and it is assumed that $C_{\text {In }}$ is proportional to $\exp \left(-x^{2} / 4 D t\right)$, where $x$ is the distance of diffusion, $D$ is the diffusion coefficient and $t$ is the annealing time. Using the convention that the diffusion length is equal to $\sqrt{4 D t}$, the In-Ga interdiffusion lengths are determined from a linear least-squares fit to the logarithm of $C_{\text {In }}$ versus distance squared (as shown in figure 10(b)). The diffusion lengths are then equal to the square roots of the negative inverse of the exponential prefactor, $1.25 \pm 0.04$ and $3.41 \pm 0.18 \mathrm{~nm}$, for the low $T$ and high $T$ annealed wetting layers, respectively. Earlier measurements of In-Ga interdiffusion lengths in this system varied by up to three orders of magnitude, from $<1$ to $>30 \mathrm{~nm}$, for a variety of groups using similar growth techniques and measurement methods [28]. Our new measurements are more precise than the earlier studies, and may be used as a guide for designing future heterostructures.

In addition to quantifying diffusion lengths, we have also estimated the activation energy for diffusion. In figure $10(c)$, the diffusion coefficients are plotted on a logarithmic scale against the reciprocal temperature. Assuming a diffusion coefficient equal to $D_{0} \exp \left(-E_{\mathrm{A}} / k T\right)$, a linear least-squares fit results in an activation energy, $E_{\mathrm{A}}=1.9 \mathrm{eV}$ and prefactor, $D_{0}=10^{-7} \mathrm{~cm}^{-2} \mathrm{~s}^{-1}$. These compare favourably with reports from the literature of activation energies, which range from 1.22 to $4.5 \mathrm{eV}$ [28].

In the plots of figure $10(a)$, a tail in the low $T$ annealed $f_{\text {In }}$ is observed along the growth direction, presumably due to surface segregation of In occurring during growth. Assuming
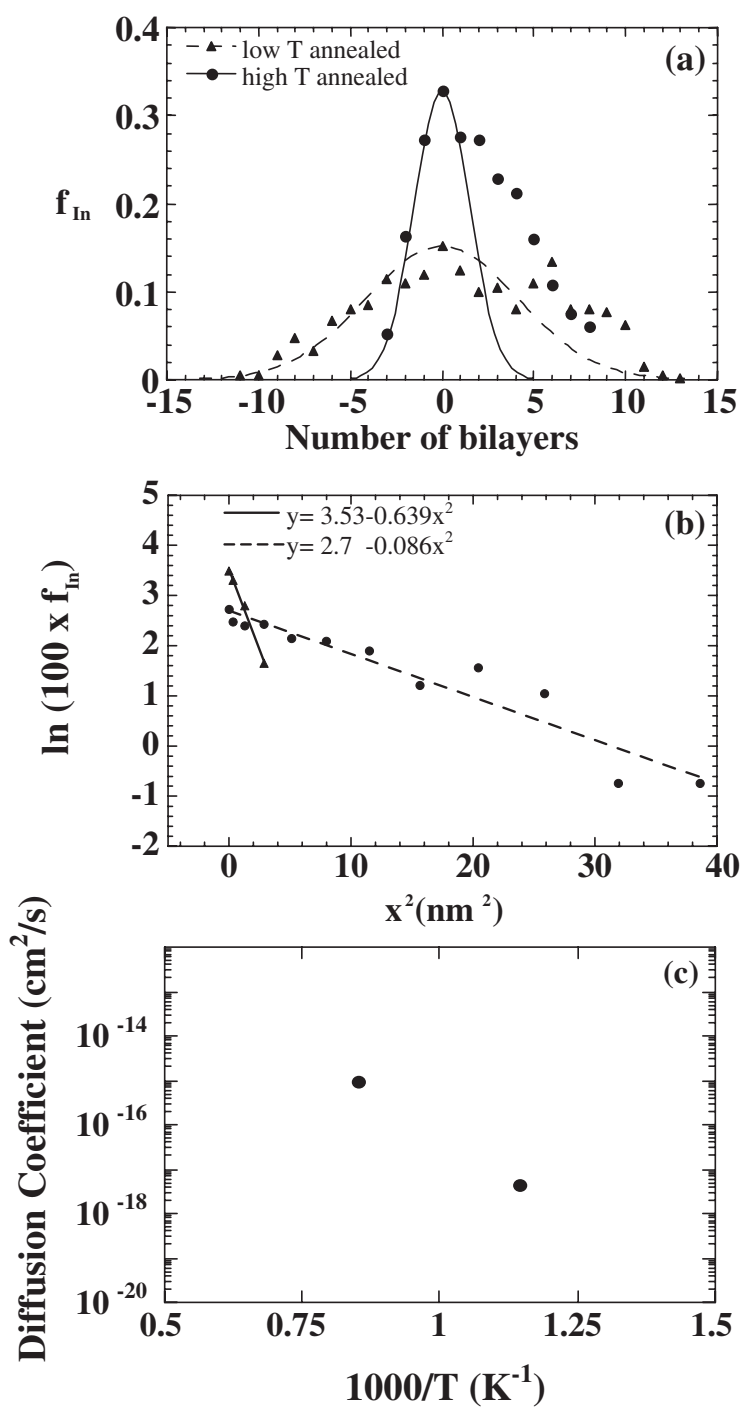

Figure 10. (a) Fraction of sites occupied by indium atoms, $f_{\mathrm{In}}$, as a function of the number of bilayers along the growth direction. (b) The concentration of indium atoms, $C_{\text {In }}=100 \times f_{\text {In }}$, on a logarithmic scale plotted against distance squared, fitted with a straight line, for the low $T$ and high $T$ annealed wetting layers. (c) Diffusion coefficient plotted against reciprocal temperature. 
that $f_{\text {In }}$ decreases exponentially along the growth direction, the distance at which $f_{\text {In }}$ decreases to $1 / \mathrm{e}$ of its initial value (the $1 /$ e segregation length) is $2.8 \pm 0.4 \mathrm{~nm}$. Although this value is similar to a number of reports of 1 /e segregation lengths for similar growth conditions, a few groups have reported larger or smaller values [28], presumably due to differences in growth conditions and characterization methods limited by averaging effects. For the high $T$ annealed wetting layer, $f_{\text {In }}$ is more symmetric about the origin, suggesting that diffusion is the dominant process determining interface broadening. Furthermore, since the high $T$ annealed diffusion length is greater than the $1 / \mathrm{e}$ segregation length discussed earlier $(2.8 \mathrm{~nm})$, it is not possible to determine the $1 / \mathrm{e}$ segregation length in that case.

\subsection{Lateral In segregation}

In the past few years, several theoretical and experimental efforts have elucidated non-randomness phenomena such as clustering, ordering and segregation in III-V semiconductor alloys [26, 27,29-34]. In InGaAs alloys, these issues are beginning to be explored in non-dilute alloys far beyond the percolation limit, such as the wetting layers of buried InAs/GaAs QDs. Here, we discuss lateral In-In pair correlations within the wetting layers, which enable examination of the applicability of Fick's first law to the nondilute InGaAs alloy system. For this purpose, we determined lateral In-In pair correlations within the wetting layers. Using experimentally determined $\mathrm{In}$ and $\mathrm{Ga}$ atom positions, we counted the number of In-In pairs, $N_{\text {In-In }}$, along the [110] direction, as a function of pair spacing, producing the open and filled circles in the plots in figures $11(a)$ and $(b)$, for the low $T$ and high $T$ annealed wetting layers, respectively. For comparison, random In-In pair distributions were generated as follows. Within each bilayer, we determined the fraction of sites occupied by In atoms and distributed them randomly. Using these computer-generated random distributions of In atoms, we then counted the number of In-In pairs along the [110] direction, as a function of spacing between them. We generated more than 4000 random distributions and averaged the number of In-In pairs to produce $N_{\text {In-In }}($ random), shown as the dashed and solid lines in figures 11(a) and (b), respectively.

For both the low $T$ and high $T$ annealed wetting layers, $N_{\text {In-In }}$ and $N_{\text {In-In }}$ (random) decrease with increasing In-In pair separation. These decreases are due in part to the finite sizes of the images examined. The pair separation beyond which $N_{\text {In-In }}\left(\right.$ random) surpasses $N_{\text {In-In }}$ increases from 6 lattice sites for the low $T$ annealed wetting layers to 17 lattice sites for the high $T$ annealed wetting layers, indicating more significant lateral clustering after high $T$ annealing. In alloys, shortrange deviations from randomness are often described by a clustering parameter, $\beta$, whereby the sign of $\beta$ indicates the tendency for clustering $(\beta>0)$ or anticlustering $(\beta<0)$ [35]. For InGaAs and related compound semiconductor alloys, $\beta$ is positive, indicating that clustering is the equilibrium state. Thus, our observation of increased In-In pair correlations with annealing suggests that the system is being driven towards its equilibrium clustered state.

The increase in In-In pair correlations with annealing also implies that vertical In-Ga interdiffusion occurs
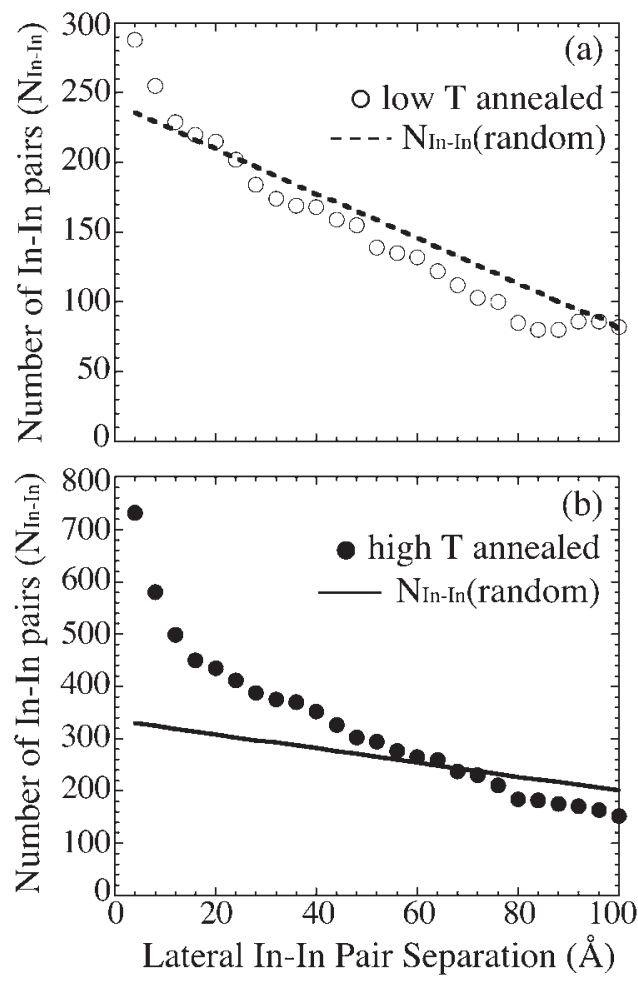

Figure 11. Plot of number of In-In pairs against In-In pair separation of $(a)$ low $T$ and $(b)$ high $T$ annealed wetting layers. The dashed line in $(a)$ and the solid line in $(b)$ represent the number of In-In pairs for the computer-generated random distributions of In atoms for the low $T$ and high $T$ annealed wetting layers, respectively. (Reprinted from [17].)

simultaneously with lateral In segregation. Fick's first law describes a diffusional flux driven solely by a concentration gradient, without contributions from additional potential gradients. In our case, the diffusional flux needs to be modified to include a term due to lateral In-In segregation, which is dependent on the In-In pair interaction energy, $E_{\text {int }}$, as follows:

$$
E_{\text {int }}=-k T \ln \left[\frac{N_{\text {In-In }}}{N_{\text {In-In }}(\text { random })}\right],
$$

where $k$ is the Boltzmann constant and $T$ is the absolute temperature [34]. Using the $N_{\text {In-In }}$ and $N_{\text {In-In }}$ (random) from figures $11(a)$ and $(b)$, we calculated $E_{\text {int }}$ as a function of In-In pair separation. We note that when $E_{\text {int }}=0$, the In-In pair interactions are negligible, and Fick's first law is considered to describe the diffusional flux of the system. In figure 12, we plot $E_{\text {int }}$ as a function of In-In pair separation, and compare these data with previous experimental and theoretical studies of dilute InGaAs alloys. In the plot, the positive and negative values of $E_{\text {int }}$ indicate repulsive and attractive interactions between neighbouring In atoms. For both the low $T$ and high $T$ annealed wetting layers, $E_{\text {int }}$ oscillates with a period of $\sim 4-5$ lattice sites. The oscillation is likely related to the periodicity of the surface reconstruction during growth discussed earlier.

In the case of low $T$ annealed wetting layers, $E_{\text {int }}$ is negative for the first few nearest-neighbours, but increases to zero for the third to sixth nearest-neighbours, and finally oscillates about a positive value for nearest-neighbours beyond 


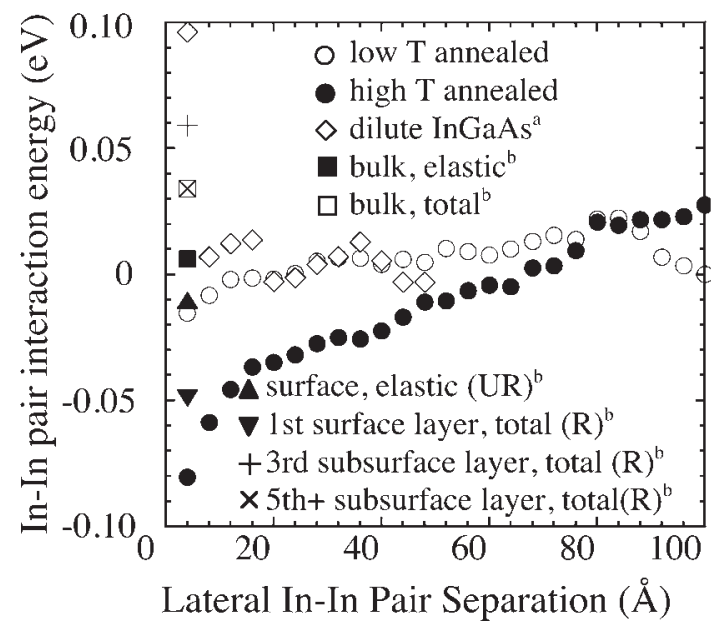

Figure 12. Plot of In-In pair interaction energy against In-In pair separation. Both experimentally and theoretically determined interaction energies for dilute InGaAs alloys are also shown. The calculated values denoted by 'total' include both elastic and chemical effects; ' $R$ ' and 'UR' refer to reconstructed and unreconstructed surface structures, respectively. (Reprinted from [17].) ${ }^{\mathrm{a}} \mathrm{See}[34] .{ }^{\mathrm{b}} \mathrm{See}[32]$.

the seventh. The significant decrease in attractive In-In pair interactions with In-In pair separation suggests the presence of strong short-range In segregation but weak long-range In-In repulsion. For comparison, an earlier experimental study of dilute InGaAs alloys, with In composition $\sim 5 \%$, is also shown in the same plot. In that case, a strong repulsive first nearest-neighbour In-In pair interaction is evident. Beyond the first nearest-neighbour, a similar weak, oscillatory repulsive interaction occurs. Thus, in the dilute InGaAs alloys, there is a strong short-range In-In repulsion and a weak long-range InIn repulsion. On the other hand, in the high $T$ annealed wetting layers, $E_{\text {int }}$ is negative out to the sixteenth nearest-neighbour In pair, suggesting the presence of both short- and long-range In segregation.

For the low $T$ annealed and high $T$ annealed wetting layers, the nearest-neighbour In-In pair interaction energies, $E_{\text {int }}(\mathrm{NN})$, are $-0.015 \mathrm{eV}$ and $-0.081 \mathrm{eV}$, respectively. Interestingly, $E_{\text {int }}(\mathrm{NN})$ for the low $T$ annealed wetting layers lies within $5 \mathrm{meV}$ of that calculated for an unreconstructed InGaAs alloy surface [32]. This similarity in values of measured and computed $E_{\text {int }}(\mathrm{NN})$ suggests that initial lateral In clustering in the InAs wetting layer may be frozen at the surface during growth. Furthermore, the more negative value of $E_{\text {int }}(\mathrm{NN})$ for the high $T$ annealed wetting layers in comparison with the low $T$ annealed wetting layers suggests a stronger attractive interaction between the In atoms in that case. As mentioned earlier, the increase in attractive In-In interactions with annealing suggests that annealing drives the system towards its equilibrium state of clustering.

\subsection{Summary and conclusions}

We have used the regions located laterally between the island arrays (i.e. the wetting layers) to directly measure indiumgallium interdiffusion and indium segregation lengths. We have also quantified the number of In-In pairs as a function of the In-In pair spacing along the [110] direction. The
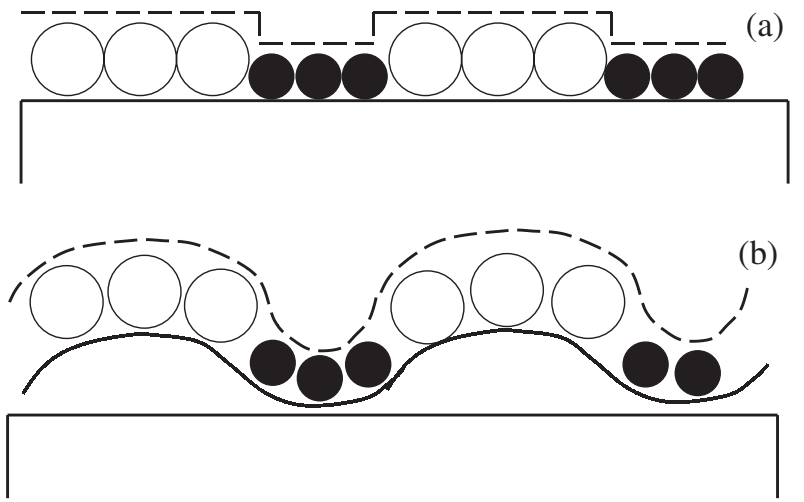

Figure 13. Schematic of possible mechanisms for initiation of phase separation. In $(a)$, compositional non-uniformities are depicted by regions with smaller dark circles and larger open circles. In $(b)$, both morphological undulations and compositional non-uniformities are apparent.

experimentally determined number of In-In pairs exceeds that of a randomly generated distribution, suggesting the presence of significant lateral In clustering within the wetting layers. Interestingly, the nearest-neighbour In-In pair interaction energies are similar to those calculated for InGaAs alloy surfaces. Since the In-In pair correlations increase with high-temperature annealing, lateral In segregation occurs simultaneously with In-Ga interdiffusion, as the system is driven towards its segregated equilibrium state.

\section{Alloy phase separation}

\subsection{Introduction}

Recently, low-dimensional semiconductor structures have been achieved by alloy decomposition of heteroepitaxial films. For example, in an alloy film AB or a SL A/B/A/B, spontaneous lateral phase separation often leads to the formation of lateral SLs consisting of alternating A-rich and B-rich layers. The relative roles of morphological undulations and nanoscale compositional non-uniformities in the initiation of alloy phase separation are the subject of continued debate [36]. For example, randomly generated nanoscale compositional nonuniformities (as shown in figure 13(a)) might lead to the development of larger-scale morphological undulations, such as those shown in figure $13(b)$, which would in turn further drive the phase separation process. Alternatively, largerscale morphological undulations shown in figure 13(b) might develop first, and the resulting significant lateral variation in strain field might lead to the nucleation of lateral compositional variations.

To date, XSTM has revealed the presence of lateral composition variations both in the absence and presence of undulations of the growth front. For example, in the InGaAsP system, lateral composition variations were observed, apparently in the absence of morphological undulations [37]. Similar effects have been observed in various mixed anion semiconductor heterostructures [38-41]. On the other hand, in the InGaAsP/InGaP SL system, XSTM and scanning tunnelling spectroscopy (STS) suggested that surface undulations are initially driven by lateral variations 
in composition, due to thickness variations in the constituent layers of the SL [42]. In that case, the growth front undulations were observed first in a strained layer, and the undulations were attributed to the elastic relaxation of strain. A similar effect, leading to the formation of vertically anticorrelated wires, was recently reported in InAs/GaSb SLs [43].

In this section, we discuss recent XSTM investigations of alloy phase separation, in heteroepitaxial films and SL structures. We consider the relative roles of morphological undulations and compositional non-uniformities on the initiation and evolution of phase separation. In the InAlAs alloy system, we show that phase separation is a misfitdriven process initiated by random compositional uniformities which later develop into coupled compositional and surface morphological variations. In the $\mathrm{ZnSnP}_{2} / \mathrm{GaAs}$ system, we reveal the presence of compositional variations which are not apparently correlated with surface morphological undulations. Finally, in the GaP/InP SPS system, we present the first direct evidence that phase separation is initiated at the onset of SPS growth. We also show that the phase separation process may be enhanced by antisite vacancy diffusion.

\subsection{Experiment}

The InAlAs samples were grown on (001)-oriented $\mathrm{p}^{+}$InP substrates, using metal-organic chemical vapour deposition at a pressure of $100 \mathrm{mbar}$, with trimethylindium (TMIn), trimethylaluminum (TMAl), $\mathrm{AsH}_{3}$ and $\mathrm{PH}_{3}$ as source gases. The targeted structures included 10 periods of $\mathrm{Zn}$-doped InAlAs/InP SLs consisting of alternating $20 \mathrm{~nm} \mathrm{InP} \mathrm{and}$ $20 \mathrm{~nm}$ InAlAs grown at $620^{\circ} \mathrm{C}$, followed by $500 \mathrm{~nm}$ of undoped InAlAs grown at $500{ }^{\circ} \mathrm{C}$. The entire structure was capped with $200 \mathrm{~nm} \mathrm{InP}$ grown at $620^{\circ} \mathrm{C}$. We will refer to the InAlAs/InP SLs as 'vertical SL' and the $500 \mathrm{~nm}$ InAlAs layers as 'bulk-like InAlAs' [44].

High-resolution x-ray rocking curves (XRC) and XRSMs were measured with a Bede D1 x-ray diffractometer using $\mathrm{Cu} \mathrm{K} \alpha$ radiation. Analysis of a series of (004) and (224) XRCs indicates that the vertical SLs are lattice-matched to the InP substrate while the bulk-like InAlAs has an average In concentration of $71 \%$, and a misfit of $1.2 \%$ with respect to the InP substrate.

The $\mathrm{ZnSnP}_{2} / \mathrm{GaAs} \mathrm{SLs}$ were grown by gas-source $\mathrm{MBE}$ using solid group III sources, and $\mathrm{AsH}_{3}$ and $\mathrm{PH}_{3}$ as gas sources. The unintentionally doped heterostructures were grown on epi-ready (001)-oriented $\mathrm{n}^{+} \mathrm{Si}$-doped GaAs substrates, with a substrate temperature of $350{ }^{\circ} \mathrm{C}$ and a $\mathrm{Sn} / \mathrm{Zn}$ flux ratio of approximately 0.1 . The targeted structures included 10-period SLs capped with $50 \mathrm{~nm}$ of GaAs. The SLs consisted of $20 \mathrm{~nm}$ $\mathrm{ZnSnP}_{2}$ quantum wells and $20 \mathrm{~nm} \mathrm{GaAs}$ barriers.

The GaP/InP SPS structures were grown on (001)-oriented $\mathrm{n}^{+}$GaAs substrate using MBE, as described elsewhere [45]. One SPS structure included 208 pairs of alternating 1.7 ML $\mathrm{GaP}$ and 1.7 ML InP layers, and the other included 659 pairs of alternating $1 \mathrm{ML} \mathrm{GaP}$ and $1 \mathrm{ML}$ InP. The SPS structures were grown at $490^{\circ} \mathrm{C}$, and sandwiched between a buffer consisting of $100 \mathrm{~nm} \mathrm{Si-doped} \mathrm{GaAs}$ grown at $570{ }^{\circ} \mathrm{C}$ and a cap consisting of at least $300 \mathrm{~nm} \mathrm{Si-doped} \mathrm{InGaP}$ grown at $490^{\circ} \mathrm{C}$. We will refer to the $(\mathrm{GaP})_{1.7} /(\mathrm{InP})_{1.7}$ SPS system as '1.7 ML SPS' and the $(\mathrm{GaP})_{1} /(\mathrm{InP})_{1}$ SPS system as ' 1 ML SPS' [46].

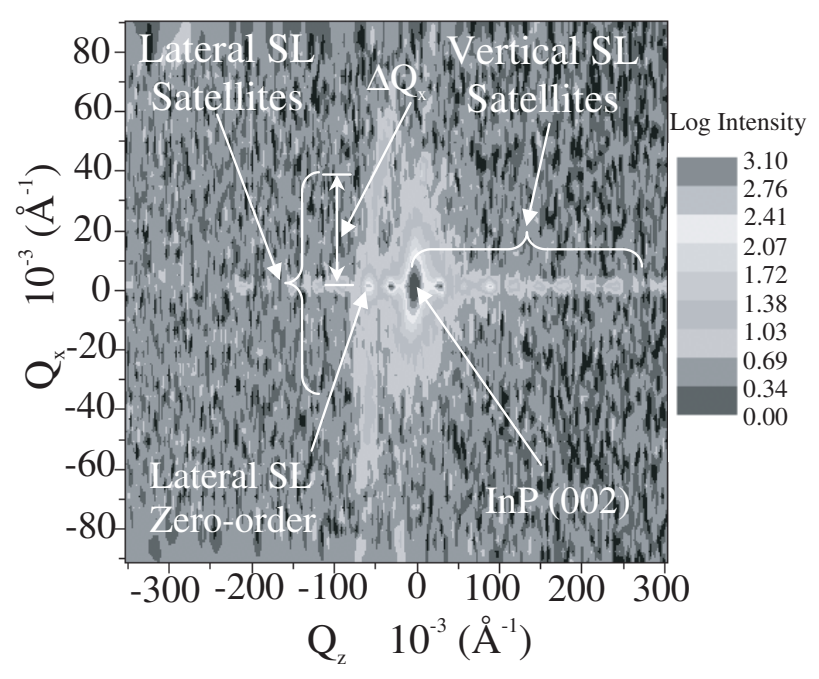

Figure 14. X-ray reciprocal space map of InAlAs/InP heterostructure collected near the $\operatorname{InP}(002)$ reflection. (Reprinted from [44].)

\subsection{InAlAs films}

An XRSM collected near the $\operatorname{InP}(002)$ reflection, with the $Q_{z}$ and $Q_{x}$ axes aligned parallel to the [001] and [110] directions, is shown in figure 14. Near the centre of the map, the $\operatorname{InP}(002)$ and surrounding satellite peaks of the vertical SL are apparent. Left of the InP substrate peak, a peak corresponding to the bulklike InAlAs is surrounded by first order satellites of a lateral SL. Using the spacing between the peak associated with the bulklike InAlAs and the first order satellite peaks of the lateral SL, $\Delta Q_{x}$, we find a modulation wavelength, $\lambda_{\text {mod }}=2 \pi / \Delta Q_{x}$, or lateral SL period of $17 \pm 1 \mathrm{~nm}$. Since these satellite peaks are present in (002) but not in (004) XRSMs, the lateral SL is primarily due to chemical or compositional variations, presumably resulting from alloy phase separation occurring at the surface during growth.

Both the vertical SL and the bulk-like InAlAs are evident in the large-scale XSTM image in figure 15(a). The InAlAs layers within the vertical SL contain isotropic clusters with typical diameters of $\sim 2 \mathrm{~nm}$ and separations ranging from 5 to $10 \mathrm{~nm}$, similar to an earlier report [29]. The bulk-like InAlAs contains quasi-periodic contrast modulations perpendicular to the growth direction. Since the lateral contrast modulations are present in the lattice-mismatched bulk-like InAlAs, but not in the misfit-free InAlAs layers within the lattice-matched vertical SL, their formation is probably related to the relaxation of misfit strain.

To investigate the initiation and evolution of the lateral contrast modulations, line-cuts from figure 15(a) were collected at various distances from the vertical SL/bulk-like InAlAs interface (as shown in figure $15(b)$ ). The tip height variations in the line-cuts, which correspond to contrast modulations in the XSTM images, are due to a combination of lateral chemical variations resulting from alloy phase separation and surface distortions resulting from elastic relaxation of strain occurring during cleaving [42]. Average modulation spacings, or apparent contrast modulation wavelengths, $\lambda$, were determined from positions throughout the thickness of the bulk-like InAlAs. Figure 16 shows $\lambda$ values 


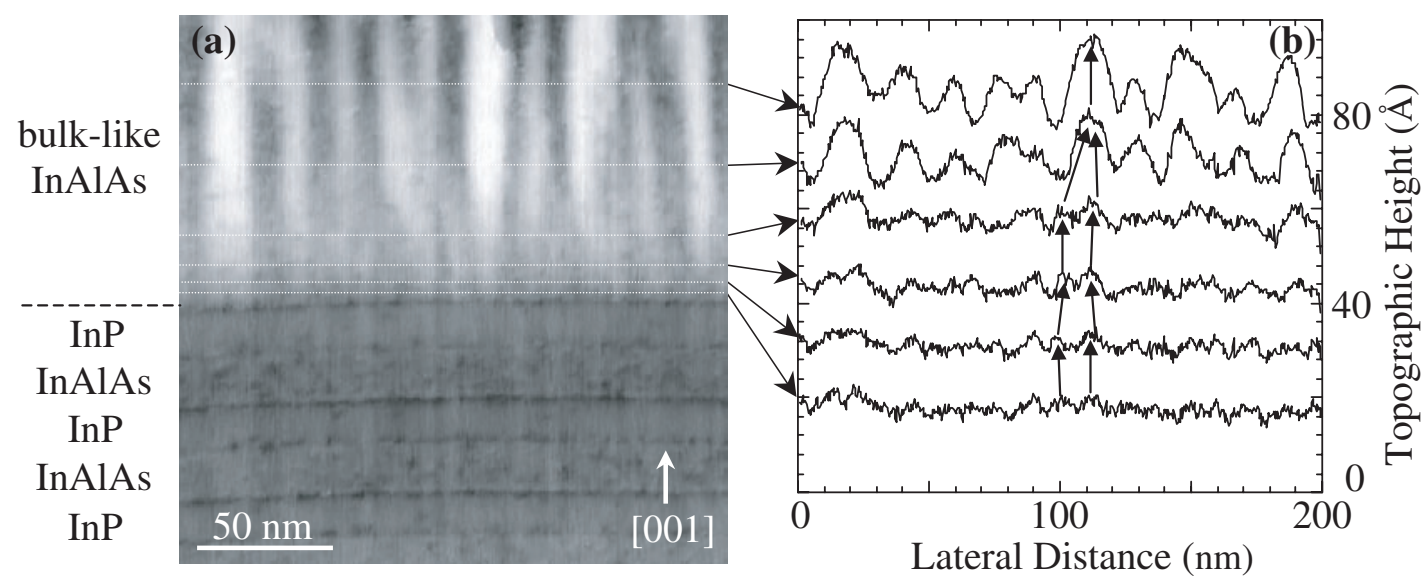

Figure 15. (a) Large-scale XSTM image showing both InAlAs/InP vertical SLs and bulk-like InAlAs, exhibiting lateral SLs. The image was acquired at a sample bias voltage of $-2 \mathrm{~V}$, and the grey-scale range displayed is $20 \AA$. Cuts of the tip height along various lines in $(a)$ are plotted in $(b)$. The merging of a pair of peaks is indicated by vertical arrows in $(b)$. (Reprinted from [44].)

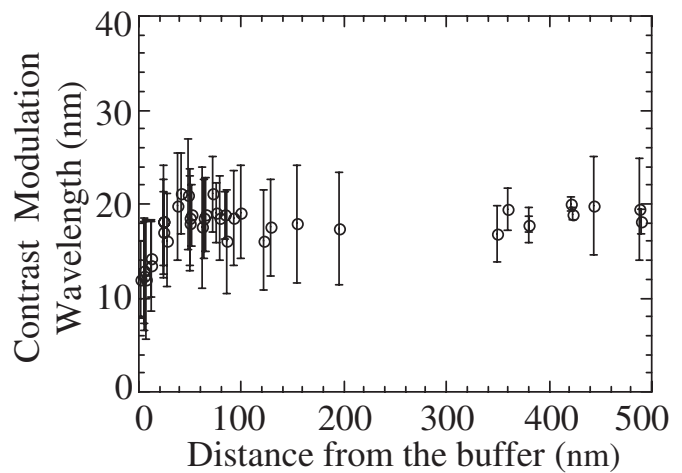

Figure 16. Plot of contrast modulation wavelength against distance from the interface between the vertical SL and the bulk-like InAlAs. (Reprinted from [44].)

from several large-scale XSTM images, plotted as a function of distance from the interface between the vertical SL and the bulk-like InAlAs. We note that in determining $\lambda$ values, only those features in line-cuts such as in figure $15(b)$, with heights at least twice the typical noise level $(\sim 0.4 \AA)$, were considered. It is evident that $\lambda$ increases with film thickness, reaching a saturation value of $\sim 20 \mathrm{~nm}$ at a film thickness $\sim 25 \mathrm{~nm}$. This saturation value of $\lambda$ is within $13 \%$ of $\lambda_{\bmod }$ determined from the (002) XRSM, suggesting that $\lambda$ in XSTM images corresponds to the wavelength of lateral composition variations.

Interestingly, the quasi-periodic lateral contrast modulations are observed in the line-cuts within only a few bilayers of the vertical SL/bulk-like InAlAs interface. Furthermore, the average modulation spacings increase with film thickness, due mainly to the merging of modulations, an example of which is indicated by arrows in figure $15(b)$. As the modulations merge, the modulation amplitude increases, due mainly to an increase in elastic strain relaxation occurring during cleaving.

An XSTM image in the vicinity of the interface between the bulk-like InAlAs and the InP cap is shown in figure 17. In the lower region of the image, lateral contrast modulations within the bulk-like InAlAs are apparent. The lateral contrast modulations persist up to a cleavage-induced surface step, whose shape resembles a morphological undulation. If the

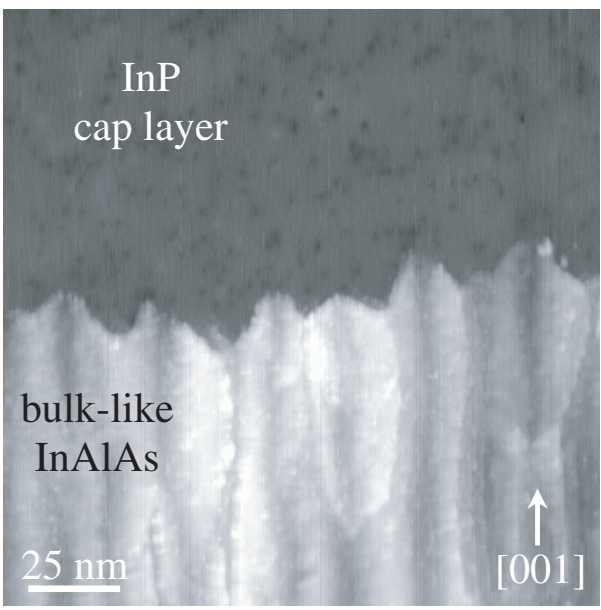

Figure 17. Large-scale XSTM topographic image at the interface between the InP cap layer and the bulk-like InAlAs, acquired at a sample bias voltage of $-1.8 \mathrm{~V}$. The grey-scale range displayed is $24 \AA$. (Reprinted from [44].)

position of the cleavage-induced surface step coincides with the interface between the bulk-like InAlAs and the InP cap, the growth front has developed a morphological undulation, with crests and troughs correlated with the lateral contrast modulations.

Modulation wavelengths of the same order of magnitude have been reported for similar films with significantly smaller misfits [47]. For phase separation initiated by morphological undulations, classical instability theory predicts an undulation wavelength inversely proportional to the misfit squared $[48,49]$. However, the fact that this proportionality is not observed experimentally suggests that alternative models need to be considered. Therefore, we propose a model in which phase separation is initiated by random compositional nonuniformities which eventually lead to the development of surface undulations.

As shown in figure 18, random compositional nonuniformities occur during the early stages of film growth (phase I). In phase II, as more layers are deposited, the phase-separated clusters merge together to more effectively relax misfit strain. Consequently, the spacing between 

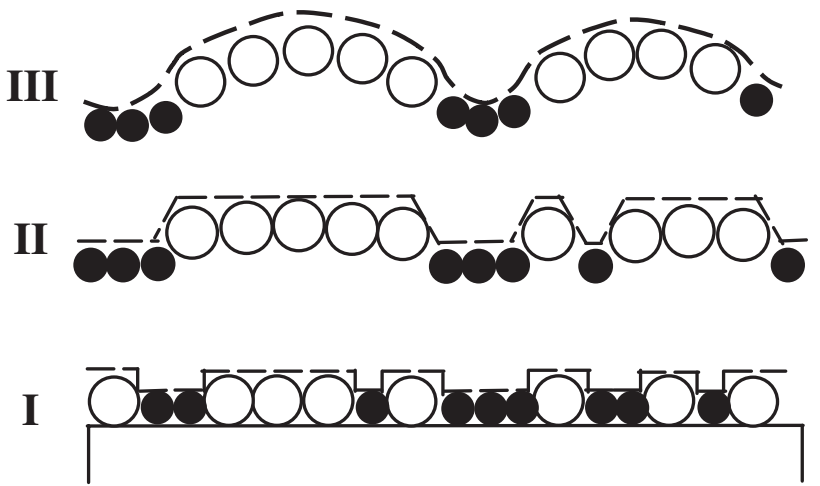

Figure 18. Schematic of proposed model for initiation and evolution of phase separation, illustrating, I: initial random compositional non-uniformities, II: merging of phase separated clusters and III: development of surface undulations, which further drive the phase separation process.

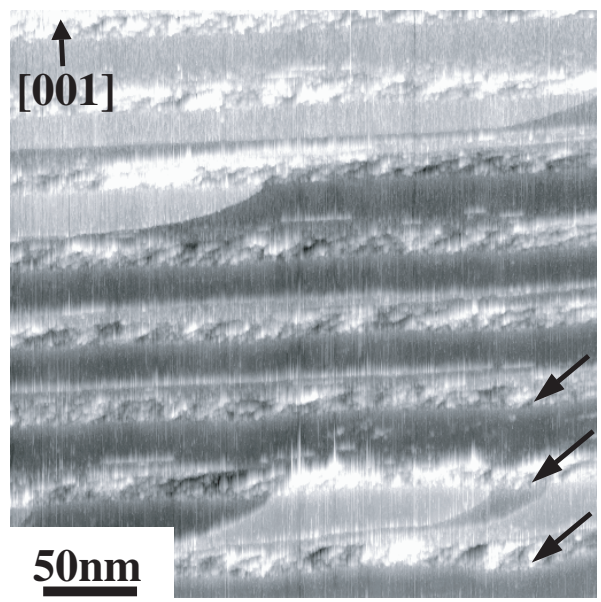

Figure 19. Large-scale XSTM topographic image of $\mathrm{ZnSnP}_{2} / \mathrm{GaAs}$ SLs, obtained at a $-2.2 \mathrm{~V}$ sample bias voltage. The grey-scale range displayed is $19 \AA$. Undulations of the growth front morphology of the $\mathrm{ZnSnP}_{2}$ on GaAs interfaces are indicated by arrows. (Reprinted from [52].)

phase-separated regions increases until it is presumably limited by surface diffusion. Meanwhile, in phase III, the merging of domains also leads to the development of surface undulations which further drive the phase separation process. Our proposed model is further supported by the fact that phase separation in our system has apparently been initiated within a few nanometres of the onset of InAlAs film growth, when planar growth is still expected. Interestingly, Spencer et al [50] recently proposed that under conditions of differing mobilities of alloying species, such as in our case where InAs is predicted to have 50 times higher surface diffusivity than AlAs [51], compositional variations may develop in the absence of surface undulations.

\section{4. $\mathrm{ZnSnP}_{2} / \mathrm{GaAs} \mathrm{SLs}$}

In $\mathrm{ZnSnP}_{2} / \mathrm{GaAs}$ SLs, XSTM and STS have revealed the presence of alloy phase separation, not apparently correlated with undulations of the growth front [52]. Figures 19 and $20(b)$ show XSTM topographic images of $\mathrm{ZnSnP}_{2} / \mathrm{GaAs}$ SLs, displayed with the growth direction from the bottom to

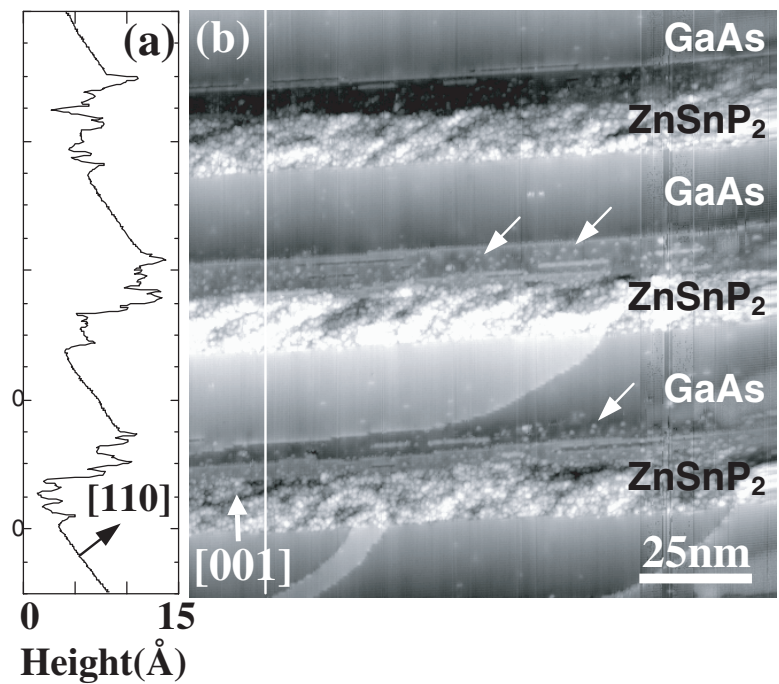

Figure 20. High-resolution XSTM topographic image of $\mathrm{ZnSnP}_{2} / \mathrm{GaAs}$ SLs, obtained with $0.15 \mathrm{nA}$ tunnelling current and $-1.8 \mathrm{~V}$ sample bias voltage. The grey-scale range displayed is $22 \AA$. A cut of the tip height along the line indicated in $(b)$ is displayed in (a). (Reprinted from [52].)

the top. In these filled state images, the $\mathrm{ZnSnP}_{2}$ regions appear as inhomogeneous layers sandwiched between homogeneous layers of GaAs. The topographic images in figures 19 and $20(b)$ are displayed at an angle with respect to the normal to the cleaved (110) surface. Hence, the cut of the tip height in figure $20(a)$, defined by the line in figure $20(b)$, reveals a cleaved surface profile that resembles a rotated staircase, with atomically flat $\mathrm{GaAs}$ regions surrounding rougher $\mathrm{ZnSnP}_{2}$ regions. Interestingly, the $\mathrm{ZnSnP}_{2}$ on GaAs interfaces are well-defined and nearly atomically abrupt, while the GaAs on $\mathrm{ZnSnP}_{2}$ interfaces are more difficult to discern due to lateral inhomogeneities in the $\mathrm{ZnSnP}_{2}$ region and the presence of a high density of cleavage steps in the vicinity of the interface.

As shown in figure 19, lateral variations in the growth surface morphology of the $\mathrm{ZnSnP}_{2}$ on GaAs interfaces are apparent. The amplitude of these growth front undulations decreases gradually towards the top of the structure, essentially disappearing after 3 to 4 SL periods. Similar types of growth front undulations have been observed in other strainedlayer SL systems $[42,43]$. In those cases, the growth front undulations were observed first in a strained layer, and the undulations were attributed to the elastic relaxation of strain. Here, the growth front undulations originate in the first GaAs layer and therefore may not be driven by elastic relaxation of strain, but rather by generic morphological instabilities occurring during homoepitaxy.

The steps and terraces observed in figure $20(b)$ are apparent in both filled and empty state topographic images (not shown), suggesting that these features are of topographic origin, possibly resulting from the cleaving process. The observed steps are generally oriented parallel to the $\mathrm{ZnSnP}_{2} / \mathrm{GaAs}$ interfaces, increasing the apparent width of the $\mathrm{ZnSnP}_{2}$ layers. Occasionally, steps located in the vicinity of the $\mathrm{GaAs}$ on $\mathrm{ZnSnP}_{2}$ interfaces are aligned up to $45^{\circ}$ away from the interface. Since most of the $45^{\circ}$ steps terminate in the $\mathrm{ZnSnP}_{2}$ layers, their formation may be 
related to significant lateral strains due to the presence of lateral compositional fluctuations in these layers. Within the $\mathrm{ZnSnP}_{2}$ layers, alternating protrusions (brighter regions) and depressions (darker regions), spanning tens of nanometres, are apparent. Since these domains appear very similarly in filled and empty state images, and the tip height difference between the domains is typically $\sim 1 \mathrm{~nm}$, it is likely that the domain contrast is dominated by topographic effects. In particular, the domain contrast is probably a cleavage-induced surface distortion resulting from significant lateral strains due to the presence of $\mathrm{ZnSnP}_{2}$ - and $\mathrm{ZnSnAs}_{2}$-rich regions. The presence of these lateral compositional fluctuations is supported by $\mathrm{x}$-ray diffraction data and STS data, shown below. Interestingly, these lateral composition fluctuations do not appear to be correlated with the growth front undulations discussed earlier.

The presence of lateral composition variations in the $\mathrm{ZnSnP}_{2}$ was further confirmed by STS measurements. In figure 21, the normalized conductance is plotted against sample

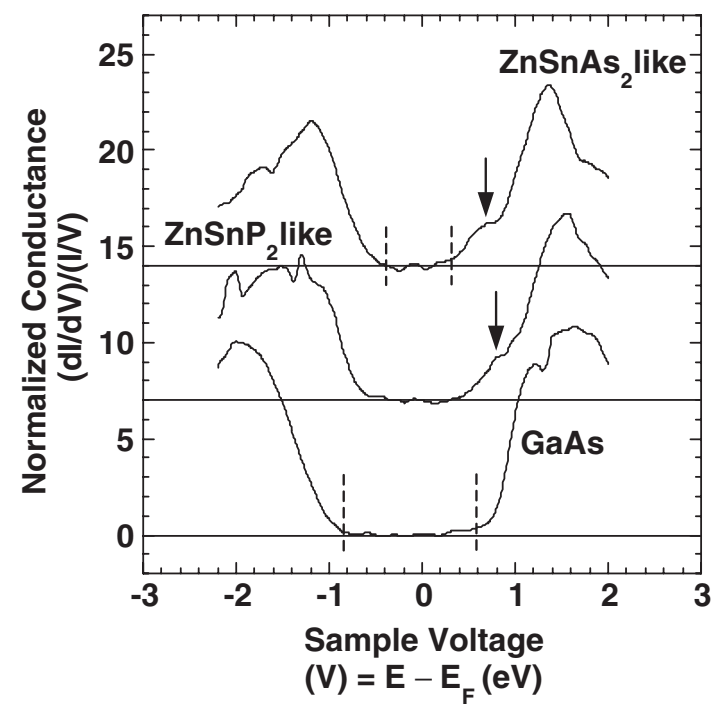

Figure 21. Normalized conductance plotted against sample bias voltage, acquired on $\mathrm{ZnSnP}_{2}$ layers, in comparison with a region of clean GaAs. The valence and conduction band edges are marked by vertical dashed lines on the left and right, respectively. A state near the conduction band edge of $\mathrm{ZnSnP}_{2}$ is indicated by downward pointing arrows. The sample voltage corresponds to the energy of the state relative to the Fermi level. (Reprinted from [52].) bias voltage for $\mathrm{ZnSnP}_{2}$ regions and compared with regions of clean GaAs. The GaAs spectrum, shown at the bottom of the figure, displays well-defined band edges, with a band gap of $1.43 \pm 0.1 \mathrm{eV}$, comparable to that of bulk GaAs. In the $\mathrm{ZnSnP}_{2}$ regions, $10-20 \mathrm{~nm}$ spaced lateral variations in the effective band gaps are observed and are attributed to alternating regions of disordered $\mathrm{ZnSnP}_{2}$ and $\mathrm{ZnSnAs}_{2}$, whose bulk band gaps are $1.24 \mathrm{eV}$ and $0.75 \mathrm{eV}$, respectively. This explanation is further supported by room temperature photoluminescence measurements which show a broad peak centred at approximately $1 \mathrm{eV}$.

\subsection{GaP/InP SPS}

Large-scale XSTM images of 1.7 ML SPS and 1 ML SPS are shown in figures $22(a)$ and $(b)$, respectively. In both images, quasi-periodic lateral contrast modulations along [110] directions are apparent in the SPS regions. Since the lateral contrast modulations are observed immediately following the interface between the SPS region and the GaAs buffer layer, it is likely that the lateral modulations were initiated at the onset of SPS growth. For both SPS structures, these lateral modulations continue throughout the entire SPS region, and the modulation spacing remains essentially constant with thickness. However, the modulation spacing of the 1 ML SPS appears larger than that of the 1.7 ML SPS.

To quantify the initiation and evolution of lateral contrast modulations, line-cuts were selected at various distances from the SPS/GaAs interface, using several large-scale XSTM images such as those in figure 22 . The tip height variations in the line-cuts, which correspond to contrast modulations in the XSTM images, are due to a combination of lateral chemical variations resulting from alloy phase separation and surface distortions resulting from elastic relaxation of strain occurring during cleaving. In the earlier study of InAlAs discussed in section 5.3, we showed that such a lateral contrast modulation represents a periodic composition variation, based on a correlation between the length-scales determined from XSTM and X-ray reciprocal space maps. Thus, in both SPS structures, we determined the average modulation spacing, or apparent contrast modulation wavelength, $\lambda$, from positions throughout the thickness of the SPS region, from several largescale images spanning a total area of more than $20 \mu \mathrm{m}^{2}$. Figure 23 shows a plot of $\lambda$ values as a function of distance
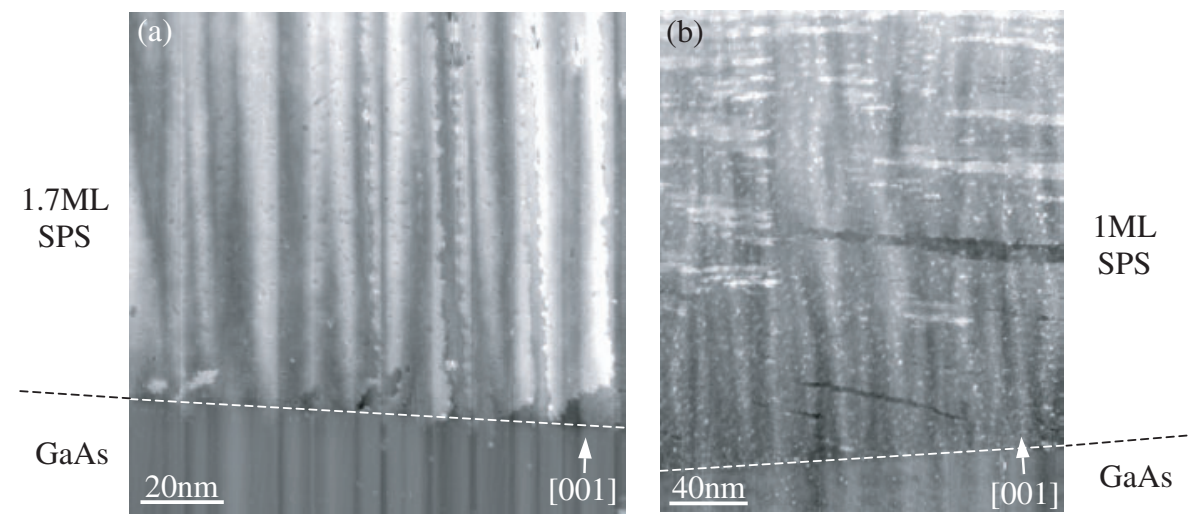

Figure 22. Large-scale XSTM topographic images showing (a) 1.7 ML SPS and $(b) 1$ ML SPS. The images were acquired at sample bias voltages of $(a)-2.5 \mathrm{~V}$ and $(b)-2.6 \mathrm{~V}$, and the grey-scale ranges displayed are $(a) 14.8 \AA$ and $(b) 10 \AA$. (Reprinted from [46].) 


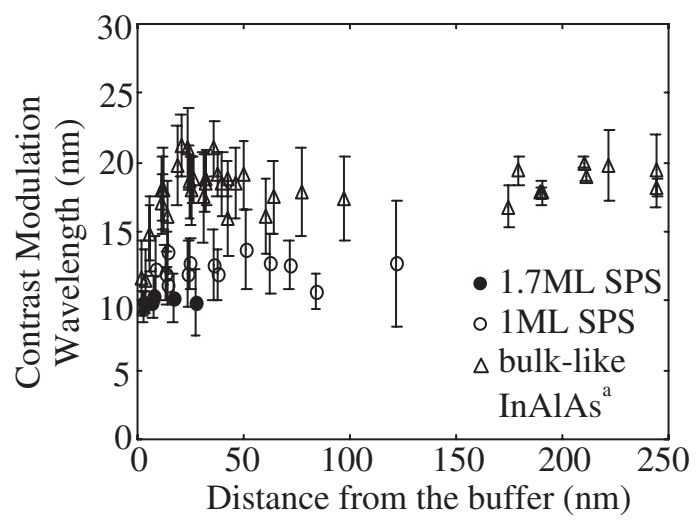

Figure 23. Plot of contrast modulation wavelength against distance from the buffer layer for 1.7 ML SPS (๑), 1 ML SPS (O), and

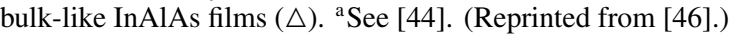

from the interface between the SPS region and the GaAs buffer layer, with filled and open circles representing $\lambda$ of $1.7 \mathrm{ML}$ and 1 ML SPS, respectively. For comparison, figure 23 also contains open triangles, indicating the $\lambda$ of bulk-like InAlAs films discussed earlier. Variations in $\lambda$ observed at particular distances from the SPS/GaAs buffer layer interface or the InAlAs/InP buffer layer interface are indicated by the error bars shown in the plot.

In the vicinity of the interface, all three $\lambda$ have a similar initial value, approximately $10 \mathrm{~nm}$. For the bulk-like InAlAs films, $\lambda$ increases with film thickness, reaching a saturation value of $\sim 20 \mathrm{~nm}$. This trend was explained by a process in which phase separation is initiated by random compositional non-uniformities occurring during the early stages of film growth. In that process, as more layers are deposited, the phase-separated regions merge together, presumably to more effectively relax misfit strain, leading to an effective increase in $\lambda$.

The spacing between phase-separated regions increases until it is presumably limited by surface diffusion, and $\lambda$ reaches a saturation value. On the other hand, for these SPS structures, $\lambda$ remains constant throughout the thickness of the entire structure. In this case, the most favourable wavelength has apparently been selected during the early stages of film growth, presumably due to a non-random distribution of compositional non-uniformities, as will be discussed below.

A high-resolution XSTM image in the vicinity of the interface between the 1.7 ML SPS and the GaAs buffer layer is shown in figure 24 . In the GaAs region, fringes with a spacing of $5.65 \AA$, corresponding to the (001) lattice planes of GaAs, are observed. In the SPS region, lateral contrast variations are observed along the [110] direction, and corresponding variations in the lattice spacings of the (001) planes are apparent. To obtain the lattice plane spacings in the regions with different contrast, we selected vertical line-cuts from both bright and dark regions within SPS structures, in several high-resolution images including figure 24. Fast Fourier transformation (FFT) analysis of the line-cuts reveals lattice spacings of 5.69 $\pm 0.01 \AA$ and $5.65 \pm 0.01 \AA$ for the bright and dark regions, respectively. Assuming a linear interpolation of binary lattice parameters (i.e. Vegard's law) and undistorted unit cells, those lattice spacings correspond to $57 \pm 3 \%$ and

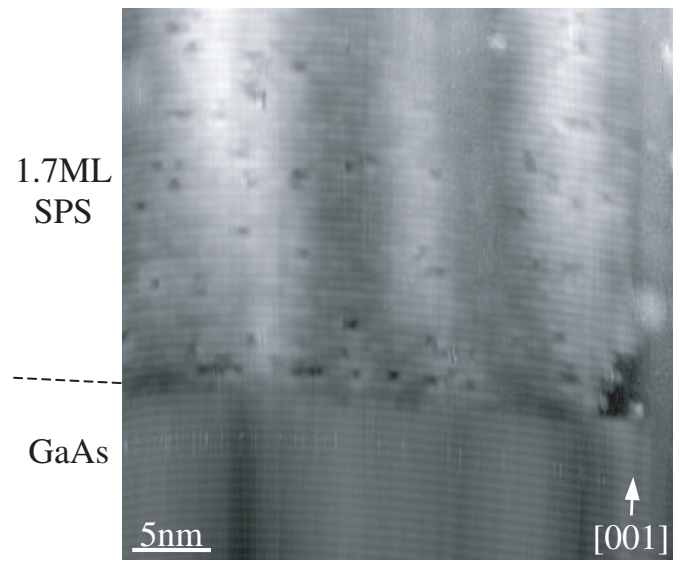

Figure 24. High-resolution XSTM image at the interface between the 1.7 ML SPS region and GaAs buffer layer, acquired at sample bias voltage of $-2.5 \mathrm{~V}$. The grey-scale range displayed is $8.4 \AA$. (Reprinted from [46].)

$48 \pm 3 \%$ In, i.e. In-rich and Ga-rich compositions, for the bright and dark regions, respectively.

In many high-resolution images such as figure 24 , a significant concentration of group $\mathrm{V}$ vacancies is observed [53]. The average vacancy concentration is $\sim 2.5 \times 10^{13} \mathrm{~cm}^{-2}$, nearly two orders of magnitude larger than that typically observed on cleaved (110) surfaces of InP [54]. Since insignificant concentrations of $\mathrm{P}$ adatoms in the SPS region, and $\mathrm{As}$ vacancies or adatoms in the GaAs region are apparent, it is not likely that the observed $\mathrm{P}$ vacancies were generated during the cleaving process. Furthermore, sequential STM images of many regions indicate negligible tip-induced vacancy generation or migration during scanning. Therefore, a significant concentration of $\mathrm{P}$ vacancies was probably incorporated into the SPS during the film growth process. These $\mathrm{P}$ vacancies may assist In-Ga interdiffusion, thereby playing a role in the phase separation process. For example, Van Vechten's calculations of antisite defect formation energies in group III-V compound semiconductors $[55,56]$ suggest that the dominant mode of vacancy diffusion is via nearest-neighbour hopping rather than hopping to secondnearest-neighbour sites on the same sublattice, implying that it is easier for an atom on a group III site to move into a vacant group III site via a vacant group V site than to jump directly into a vacant group III site [57]. Moreover, a number of experiments supporting diffusion of group III atoms via group $\mathrm{V}$ vacancies have been reported $[58,59]$. We note that phase separation in these structures was only observed for the highest growth temperatures, where $\mathrm{P}$ desorption is expected to be the most significant [45], suggesting a correlation between growth conditions resulting in high $\mathrm{P}$ vacancy concentrations and phase separation. Further work is needed to determine the precise interplay between vacancy formation, V/III ratio and alloy phase separation in this and other SPS systems.

These XSTM observations, including the differences in modulation wavelengths of the 1.7 ML and 1 ML SPS, and the modulation wavelength apparently fixed at the earliest stage of film growth, suggest a mechanism in which lateral phase separation is initiated by non-random compositional nonuniformities from excess surface adatoms due to incomplete coverage of the constituent layers of the SL, similar to the 
suggestion of Pearah et al [60]. Starting with this mechanism, we explain the dependence of $\lambda$ on the thickness of the constituent layers of the SPS, as follows. At the onset of SPS growth, excess surface adatoms, resulting from incomplete coverage of one of the constituent layers, lead to the formation of surface clusters. As the SPS is grown, the other constituent layer fills the regions between clusters, eventually forming additional clusters. Interestingly, the deviation from complete coverage is greater for the $1.7 \mathrm{ML}$ SPS than for the $1 \mathrm{ML}$ SPS, while the growth conditions are essentially identical. Thus, more clusters, and a consequently smaller initial $\lambda$, are expected for the $1.7 \mathrm{ML}$ SPS than for the $1 \mathrm{ML}$ SPS, consistent with our XSTM observations. The apparent regular spacing between the surface clusters is likely to be caused by In-Ga interdiffusion, enhanced in the presence of significant $P$ vacancies.

\subsection{Summary and conclusions}

In summary, we have used XSTM to investigate alloy phase separation in heteroepitaxial films and SLs, including InAlAs, $\mathrm{ZnSnP}_{2} / \mathrm{GaAs}$, and $\mathrm{GaP} / \mathrm{InP}$. In all cases, we consider the relative roles of compositional non-uniformities and morphological undulations in the initiation and evolution of alloy phase separation. We show that phase separation is a misfitdriven process, most often initiated by compositional nonuniformities which later develop into coupled compositional and surface morphological undulations.

\section{Overall summary and future directions}

In this paper, we described XSTM and its application to investigations of epitaxial semiconductor heterostructures, including studies of the formation and ordering of QD arrays, measurements of interdiffusion and segregation lengths, and investigations of the mechanisms of alloy phase separation. In the studies of QD arrays (discussed in section 3) we presented a new model for ordering of QDs, in which the lateral column spacing is determined by surface nucleation during growth followed by strain-enhanced island dissolution during annealing. As discussed in section 4, we used the regions located laterally between the island arrays (i.e. the 'wetting' layers), to directly measure In-Ga interdiffusion and In segregation lengths, and to reveal that lateral In segregation occurs simultaneously with vertical In-Ga interdiffusion. Finally, in section 5, we discussed the mechanisms of alloy phase separation in several heteroepitaxial films and SLs. We showed that alloy phase separation is a misfitdriven process, most often initiated by compositional nonuniformities which later develop into coupled compositional and surface morphological undulations.

Future directions of interest include interface studies for spin-electronics and spin-optoelectronics. For example, quantitative studies of alloy formation in dilute magnetic semiconductor systems, including GaMnAs and Mn-doped InAs/GaAs QDs, are needed. In particular, distributions of Mn atoms and other defects need to be quantified and correlated with the resulting magnetic properties, in order to optimize these materials for near or above room temperature operation. An additional area of future interest is XSTM studies of (110)-oriented heterostructures, for which spin-injection is optimized [61].

\section{Acknowledgments}

The author gratefully acknowledges many students and colleagues who contributed to this work, including B Lita, B Shin, W Chen, A Lin, K Lappo, J Phillips, A Stiff-Roberts and P K Bhattacharya, all from the University of Michigan; as well as G Seryogin (Texas Tech.), S A Nikishin (Texas Tech.), H Temkin (Texas Tech.), M C Hanna (NREL), S Francoeur (NREL), A G Norman (NREL), A Mascarenhas (NREL), J D Song (K-JIST), J M Kim (K-JIST) and Y T Lee (K-JIST). The work at University of Michigan was supported in part by the Dow-Corning Foundation, the Petroleum Research Fund, the National Science Foundation, the Army Research Office and the Office of Naval Research.

\section{References}

[1] Feenstra R M 1994 Semicond. Sci. Technol. 92157

[2] Yu E T 1996 Mater. Sci. Eng. R 17147

[3] Albrektson O 1990 PhD Thesis Technical University, Denmark

[4] Yu E T 1997 Chem. Rev. 971017

[5] Lutz M A, Feenstra R M and Chu J O 1995 Surf. Sci. 328215

[6] Nuffer R, Mussig H-J and Dabrowski J 2000 Solid-State Electron. 44875

[7] Feenstra R M 1994 Phys. Rev. B 504561

[8] Eisele H, Flebbe O, Kalka T and Dahne-Prietsch M 1999 Surf. Interface Anal. 27537

[9] Legrand B, Grandidier B, Nys J P, Stievenard D, Gerard J M and Thierry-Mieg V 1998 Appl. Phys. Lett. 7396

[10] Liu N, Tersoff J, Baklenov O, Holmes A L and Shih C K 2000 Phys. Rev. Lett. 84334

[11] Liu N, Lyeo H K, Shih C K, Oshima M, Mano T and Koguchi N 2002 Appl. Phys. Lett. 804345

[12] Solomon G S, Wu W, Tucker J R and Harris J S 1998 Physica $\mathrm{E} 2709$

[13] Wu W, Tucker J R, Solomon G S and Harris J S 1997 Appl. Phys. Lett. 711083

[14] Lita B, Goldman R S, Phillips J D and Bhattacharya P K 1999 Appl. Phys. Lett. $\mathbf{7 5} 2797$

[15] Lita B, Goldman R S, Phillips J D and Bhattacharya P K 1999 Appl. Phys. Lett. $\mathbf{7 4} 2824$

[16] Lita B, Goldman R S, Phillips J D and Bhattacharya P K 2000 Surf. Rev. Lett. 7539

[17] Shin B, Lita B, Goldman R S, Phillips J D and Bhattacharya P K 2002 Appl. Phys. Lett. 811423

[18] Xie Q, Madhukar A, Chen P and Kobayashi N 1995 Phys. Rev. Lett. 752542

[19] Tersoff J, Teichert C and Lagally M G 1996 Phys. Rev. Lett. 75 2542

[20] Thanh V, Yam V and Nguyen L H 2002 J. Vac. Sci. Technol. B 201259

[21] Holy V, Springholz G, Pinczolits M and Bauer G 1999 Phys. Rev. Lett. 83356

[22] Mateeva E, Sutter P, Bean J C and Lagally M G 1997 Appl. Phys. Lett. 713233

[23] Xu S J, Wang H, Li Q, Xie M H, Wang X C, Fan W J and L F W 2000 Appl. Phys. Lett. 742824

[24] Chen W, Shin B, Goldman R S, Stiff A and Bhattacharya P K 2003 J. Vac. Sci. Technol. B 211920

[25] Migliorato M A, Wilson L R, Mowbray D J, Skolnick M S Al-Khafaji M, Cullis A G and Hopkinson M $2001 \mathrm{~J}$. Appl. Phys. 906374

[26] Zheng J F, Walker J D, Salmeron M B and Weber E R 1994 Phys. Rev. Lett. 722414 
[27] Pfister M, Johnson M B, Alvarado S F, Salemink H W M, Marti U, Martin D, Morier-Genoud F and Reinhart F K 1995 Appl. Phys. Lett. 671459

[28] Lita B 2002 PhD Thesis University of Michigan

[29] Seaford M L, Wu W, Eyink K G, Tomich D H, Tucker J R and Eastman L F 1997 J. Vac. Sci. Technol. B 151274

[30] Kawai T, Yonezu H, Ogasawara Y, Saito D and Pak K 1993 J. Appl. Phys. 741770

[31] Yamaguchi K, Yasuda Y, Kovacs A and Barna P B 2001 J. Appl. Phys. 89217

[32] Cho J-H, Zhang S B and Zunger A 2000 Phys. Rev. Lett. 84 3654

[33] McKay H A, Feenstra R M, Schmidtling T and Pohl U W 2001 Appl. Phys. Lett. 7882

[34] Chao K J, Shih C K, Gotthold D W and Streetman B G 1997 Phys. Rev. Lett. 794822

[35] Yamazaki S, Kishi M and Katoda T 1982 Phys. Stat. Sol. B 11342

[36] Zunger A and Mahajan S 1994 Handbook on Semiconductors vol 3, ed S Mahajan (Amsterdam: North-Holland) p 1399

[37] McComb D W, Okada T, Weatherly G C, Wolkow R A and Hulse J E 1996 Phil. Mag. Lett. 73129

[38] Zuo S L, Bi W G, Tu C W and Yu E T 1998 Appl. Phys. Lett. 722135

[39] Zuo S L, Bi W G, Tu C W and Yu E T 1998 J. Vac. Sci. Technol. B 162395

[40] Yu E T 1999 Micron 3051

[41] Yu E T, Zuo S L, Bi W G, Tu C W, Allerman A A and Biefeld R M 1999 J. Vac. Sci. Technol. A 172246

[42] Goldman R S, Feenstra R M, Silfvenius C, Stalnacke B and Landgren G 1997 J. Vac. Sci. Technol. B 151027

[43] Nosho B Z, Bennett B R, Whitman L J and Goldenberg M 2002 Appl. Phys. Lett. 814452
[44] Shin B, Lin A, Lappo K, Goldman R S, Hanna M C, Francoeur S, Norman A G and Mascarenhas A 2002 Appl. Phys. Lett. 803292

[45] Song J D, Ok Y-W, Kim J M, Lee Y T and Seong T-Y 2001 J. Appl. Phys. 905086

[46] Shin B, Chen W, Goldman R S, Song J D, Kim J M and Lee Y T 2004 J. Vac. Sci. Technol. B 22216

[47] Cho H K, Lee J Y, Kwon M S, Lee B, Baek J H and Han W S 1999 Mater. Sci. Eng. B 64174

[48] Srolovitz D J 1989 Acta Metall. 37621

[49] Asaro R J and Tiller W A 1972 Metall. Trans. 31789

[50] Spencer B J, Voorhees P W and Tersoff J 2000 Appl. Phys. Lett. 763022

[51] Shitara T, Vvedensky D D, Neave J H and Joyce B A 1993 Proc. Symp. Mater. Res. Soc. 312267

[52] Lita B, Beck M, Goldman R S, Seryogin G A, Nikishin S A and Temkin H 2000 Appl. Phys. Lett. 772894

[53] Ebert P, Urban K and Lagally M G 1994 Phys. Rev. Lett. 72840

[54] Ebert P, Heinrich M, Simon M, Urban K and Lagally M G 1995 Phys. Rev. B 519696

[55] Van Vechten J A 1975 J. Electrochem. Soc. 122419

[56] Van Vechten J A 1975 J. Electrochem. Soc. 122423

[57] Van Vechten J A and Wager J F 1985 J. Appl. Phys. 57 1956

[58] Juang M T, Wager J F and Van Vechten J A 1988 J. Electrochem. Soc. 1352019

[59] Laidig W D, Holonyak N, Camras M D, Hess K, Coleman J J, Dapkus P D and Bardeen J 1981 Appl. Phys. Lett. 38776

[60] Pearah P J, Chen A C, Hsieh K C and Cheng K-Y 1994 IEEE J. Quantum Electron. 30608

[61] Hall K C, Gundogdu K, Altunkaya E, Lau W H, Flatte M E and Boggess T F 2003 Phys. Rev. B 68115311 